\title{
NON-LOCAL APPROXIMATION OF FREE-DISCONTINUITY PROBLEMS WITH LINEAR GROWTH
}

\author{
LUCA LUSSARDi ${ }^{1}$ AND ENRICO VITALI ${ }^{1}$
}

\begin{abstract}
We approximate, in the sense of $\Gamma$-convergence, free-discontinuity functionals with linear growth in the gradient by a sequence of non-local integral functionals depending on the average of the gradients on small balls. The result extends to higher dimension what we already proved in the one-dimensional case.
\end{abstract}

Mathematics Subject Classification. 49Q20, 49J45, 49M30.

Received July 7, 2005. Revised November 22, 2005.

\section{INTRODUCTION}

A number of variational problems recently under consideration involves integral functionals with "free discontinuities" (according to a terminology introduced in [20]): the variable function $u$ is required to be smooth only outside a surface $K$, depending on $u$, and both $u$ and $K$ enter the structure of the functional. Hence, a typical form is:

$$
F(u, K)=\int_{\Omega \backslash K} \phi(|\nabla u|) \mathrm{d} x+\int_{K \cap \Omega} \vartheta\left(\left|u^{+}-u^{-}\right|\right) \mathrm{d} \mathcal{H}^{n-1},
$$

where $\Omega$ is an open subset of $\mathbb{R}^{n}, K$ is a $(n-1)$-dimensional compact set, $\left|u^{+}-u^{-}\right|$is the jump of $u$ across $K$, while $\phi$ and $\vartheta$ are given positive functions (and $\mathcal{H}^{n-1}$ denotes the $(n-1)$-dimensional Hausdorff measure).

The natural weak formulation is obtained looking at $K$ as the set of discontinuities of $u$, thus working in spaces of functions allowing hypersurfaces of discontinuities, such as the space $B V(\Omega)$ of functions of bounded variation.

The main difficulty in the actual minimization of $F$ is the presence of the $(n-1)$-dimensional integral: the need of suitable approximations (leading to the convergence of minimum points) by means of more manageable functionals naturally arises. The method introduced in [12], when $\phi(t)=t^{2}$ and $\vartheta$ is constant, makes use of integral functionals whose density depends on the average of the gradient on small balls. Here we apply this model to the case of $\phi$ having linear growth at infinity.

The aforementioned weak form of $F$ in $B V(\Omega)$ takes the form:

$$
F(u)=\int_{\Omega} \phi(|\nabla u|) \mathrm{d} x+\int_{S_{u}} \vartheta\left(\left|u^{+}-u^{-}\right|\right) \mathrm{d} \mathcal{H}^{n-1}+c_{0}\left|D^{c} u\right|(\Omega),
$$

Keywords and phrases. Variational approximation, free discontinuities.

1 Dipartimento di Matematica "F. Casorati" Via Ferrata 1, 27100, Pavia, Italy; luca.lussardi@unipv.it; enrico.vitali@unipv.it 
where $D u=\nabla u \mathrm{~d} x+\left(u^{+}-u^{-}\right) \mathrm{d} \mathcal{H}^{n-1}+D^{c} u$ is the decomposition of the measure derivative of $u$ in its absolutely continuous, jump and Cantor part, respectively, and $S_{u}$ denotes the set of discontinuity points of $u$. Assuming that $\phi$ is convex and $\vartheta$ is concave, with

$$
\lim _{t \rightarrow+\infty} \frac{\phi(t)}{t}=c_{0}=\lim _{t \rightarrow 0^{+}} \frac{\vartheta(t)}{t},
$$

it turns out that $F$ is lower semicontinuous with respect to the $L^{1}$-topology. Note that if $\phi$ has superlinear growth at infinity then $c_{0}=+\infty$ and $F(u)$ is finite only if $D u$ has no Cantor part (i.e. $u$ belongs to the so-called space of special functions with bounded variation). The well-known Mumford-Shah functional falls within this case: $\int_{\Omega}|\nabla u|^{2} \mathrm{~d} x+\mathcal{H}^{n-1}\left(S_{u}\right)$.

As pointed out in [12], it is not possible to obtain a variational approximation of $F$ by usual integral functionals of the form

$$
F_{\varepsilon}(u)=\int_{\Omega} f_{\varepsilon}(\nabla u) \mathrm{d} x
$$

on Sobolev spaces; indeed, passing to the lower semicontinuous envelopes, this would lead to a convex limit, which contrasts with the non-convexity of $F$.

Heuristic arguments suggest that to get around the difficulty we have to prevent the consideration or the optimality of approximation gradients which are "too large" (with respect to $1 / \varepsilon$ ), or to prevent that the effect of "large" gradients is concentrated on "small" regions. Several approximation methods fit this requirements: see, e.g., the case where the functionals $F_{\varepsilon}$ are restricted to finite elements spaces on regular triangulations of size $\varepsilon[9,13,23]$; or the implicit constraint on the gradient through the addition of a higher order penalization $[1,3,22]$; or the study of non-local models, where the effect of a "large" gradient is "spread" onto a set of size $\varepsilon$ : this is the method which was first applied to the Mumford-Shah functional by Braides and Dal Maso in [12] (see also [11,14-16,18]), and that we follow in this paper for the case of linear growth. We also have to mention the Ambrosio and Tortorelli approximation (see [6] and [7]) of the Mumford-Shah functional via elliptic functionals, where an additional variable, say $v$, which approaches the characteristic function of the complement of the discontinuity set, is introduced.

A variant of this last method was studied in $[25,26]$ and [2] for functionals with linear growth in the gradient: the attempt is to unify the curve evolution method used in Computer Vision to detect boundaries, and the pre-processing of the image to provide an "edge-strength" function $v$, which indicates the likelihood of an object boundary being present at any point of the domain (compare with the additional variable $v$ in the Ambrosio-Tortorelli functional). Indeed, in the method of shape recovery by curve evolution, we try to detect the boundaries as curves $\Gamma$ where the image intensity gradient (hence $v$ ) is high; therefore, we apply gradient descent to the functional $\int_{\Gamma}(1-v)^{2} \mathrm{~d}_{\mathcal{H}}{ }^{1}$ (see [25] and [26]). Following Osher and Setian [24] it is convenient to embed the initial curve in a surface (the graph of a function $u$ ) as a level curve, and to apply the evolution to the surface, so that all of its level curves evolve simultaneously. Hence the functional $\int_{-\infty}^{+\infty} \int_{\{u=c\}}(1-v)^{2} \mathrm{~d} \mathcal{H}^{1} \mathrm{~d} c$ is taken into account. By the coarea formula this is nothing but

$$
\int_{\Omega}(1-v)^{2}|\nabla u| \mathrm{d} x
$$

In [25] and [26] a new segmentation functional was proposed by inserting this term in place of the square-gradient term of the Ambrosio-Tortorelli functional. In [2] the limit functional is proved to be of the form (1.1), with $\phi$ satisfying (1.2).

Here we tackle the problem of the approximation of a functional $F$ as in (1.1) with $\phi$ of linear growth, by the method of non-local functionals. More precisely, we consider the problem of the convergence of

$$
F_{\varepsilon}(u)=\frac{1}{\varepsilon} \int_{\Omega} f\left(\varepsilon f_{B_{\varepsilon}(x) \cap \Omega}|\nabla u(y)| \mathrm{d} y\right) \mathrm{d} x
$$


as $\varepsilon \rightarrow 0$ (here $B_{\varepsilon}(x)$ denotes the ball of centre $x$ and radius $\varepsilon$ ). Unlike the one-dimensional case we dealt with in [21], here we restrict the study to a fixed integrand function $f$, independent of $\varepsilon$. In the limit (see Th. 3.1) the bulk and Cantor parts are completely determined by the behaviour of $f$ at 0 (namely, we get $f^{\prime}(0)\left(\left|D^{a} u\right|(\Omega)+\right.$ $\left.\left|D^{c} u\right|(\Omega)\right)$ while the surface energy density can be explicitely computed as $\vartheta(s)=2 \int_{0}^{1} f\left(\frac{\omega_{n-1}}{\omega_{n}} s\left(\sqrt{1-t^{2}}\right)^{n-1}\right) \mathrm{d} t$ (here $\omega_{k}$ denotes the volume of the $k$-dimensional ball in $\mathbb{R}^{k}$ ).

\section{Notation AND PRELIMINARIES}

Let $n \geq 1$ be a fixed integer. The scalar product of $x, y \in \mathbb{R}^{n}$ is denoted by $\langle x, y\rangle$ and the euclidean norm by $|x|$. The open ball with centre $x$ and radius $r$ is indicated by $B_{r}(x)$; the boundary of the unit ball $B_{1}(0)$ is denoted by $S^{n-1}$. The Lebesgue measure and the $(n-1)$-dimensional Hausdorff measure of a Borel set $B \subseteq \mathbb{R}^{n}$ are denoted by $|B|\left(\right.$ or $\left.\mathcal{L}^{n}(B)\right)$ and $\mathcal{H}^{n-1}(B)$, respectively. We use standard notation for Lebesgue spaces $L^{p}(\Omega)$ and Sobolev spaces $W^{1, p}(\Omega)$.

Functions of bounded variation. For a thorough treatment of $B V$ functions we refer to [5]. Let $\Omega$ be an open subset of $\mathbb{R}^{n}$. We recall that the space $B V(\Omega)$ of real functions of bounded variation is the space of the functions $u \in L^{1}(\Omega)$ whose distributional derivative is representable by a measure in $\Omega$, i.e.,

$$
\int_{\Omega} u \frac{\partial \varphi}{\partial x_{i}} \mathrm{~d} x=-\int_{\Omega} \varphi \mathrm{d} D_{i} u \quad \text { for every } \varphi \in C_{c}^{\infty}(\Omega) \text { and } i=1, \ldots, n
$$

for some $\mathbb{R}^{n}$-valued measure $D u=\left(D_{1} u, \ldots, D_{n} u\right)$ on $\Omega$. Clearly, the Sobolev space $W^{1,1}(\Omega)$ is contained in $B V(\Omega)$.

Let $u \in B V(\Omega)$. We say that $u$ has approximate limit at $x \in \Omega$ if there exists $z \in \mathbb{R}$ such that

$$
\lim _{\varrho \rightarrow 0^{+}} f_{B_{\varrho}(x)}|u(y)-z| \mathrm{d} y=0 .
$$

The set $S_{u}$ where this property fails is called approximate discontinuity set of $u$. The vector $z$ is uniquely determined for any point $x \in \Omega \backslash S_{u}$ and is called the approximate limit of $u$ at $x$ and denoted by $\tilde{u}(x)$.

We say that $x$ is an approximate jump point of the function $u \in B V(\Omega)$ if there exist $a, b \in \mathbb{R}$ and $\nu \in \mathbb{R}^{n}$ with $|\nu|=1$, such that $a \neq b$ and

$$
\lim _{\varrho \rightarrow 0^{+}} f_{B_{\varrho}^{+}(x, \nu)}|u(y)-a| \mathrm{d} y=0, \quad \lim _{\varrho \rightarrow 0^{+}} f_{B_{\varrho}^{-}(x, \nu)}|u(y)-b| \mathrm{d} y=0,
$$

where $B_{\varrho}^{+}(x, \nu)=\left\{y \in B_{\varrho}(x):\langle y-x, \nu\rangle>0\right\}$ and $B_{\varrho}^{-}(x, \nu)=\left\{y \in B_{\varrho}(x):\langle y-x, \nu\rangle<0\right\}$. The set of approximate jump points of $u$ is denoted by $J_{u}$. The triplet $(a, b, \nu)$, which turns out to be uniquely determined up to a permutation of $a$ and $b$ and a change of sign of $\nu$, is denoted by $\left(u^{+}(x), u^{-}(x), \nu_{u}(x)\right)$. On $\Omega \backslash S_{u}$ we set $u^{+}=u^{-}=\tilde{u}$.

Theorem 2.1 (Federer-Vol'pert). For any $u \in B V(\Omega)$ the set $S_{u}$ is countably $(n-1)$-rectifiable and $\mathcal{H}^{n-1}\left(S_{u} \backslash\right.$ $\left.J_{u}\right)=0$. Moreover, $D u\left\llcorner J_{u}=\left(u^{+}-u^{-}\right) \nu_{u} \mathcal{H}^{n-1}\left\llcorner J_{u}\right.\right.$, and $\nu_{u}(x)$ gives the approximate normal direction to $J_{u}$ for $\mathcal{H}^{n-1}$-a.e. $x \in J_{u}$.

For a function $u \in B V(\Omega)$ let $D u=D^{a} u+D^{s} u$ be the (Lebesgue) decomposition of $D u$ into absolutely continuous and singular part. We denote by $\nabla u$ the density of $D^{a} u$; the measures

$$
D^{j} u:=D^{s} u\left\llcorner J_{u}, \quad D^{c} u:=D^{s} u\left\llcorner\left(\Omega \backslash S_{u}\right)\right.\right.
$$

are called the jump part and the Cantor part of the derivative, respectively. Since $D u$ vanishes on $\mathcal{H}^{n-1}$ negligible Borel sets (see [5], Lem. 3.76), from the Federer-Vol'pert Theorem we can write:

$$
D u=\nabla u \mathcal{L}^{n}+\left(u^{+}-u^{-}\right) \nu_{u} \mathcal{H}^{n-1}\left\llcorner S_{u}+D^{c} u .\right.
$$


Finally, let us recall the following important compactness Theorem in $B V$ (see Th. 3.23 and Prop. 3.21 in [5]):

Theorem 2.2. Let $\Omega$ be a bounded open subset of $\mathbb{R}^{n}$ with Lipschitz boundary. Every sequence $\left(u_{h}\right)$ in $B V(\Omega)$ which is bounded in $B V(\Omega)$ admits a subsequence converging in $L^{1}(\Omega)$ to a function $u \in B V(\Omega)$.

Spaces $S B V$ and $G S B V$. Let $\Omega$ be an open subset of $\mathbb{R}^{n}$. We say that a function $u \in B V(\Omega)$ is a special function of bounded variation $(u \in S B V(\Omega))$ if $\left|D^{c} u\right|(\Omega)=0$.

We say that a function $u \in L^{1}(\Omega)$ is a generalized function of bounded variation $(u \in G B V(\Omega))$ if $u^{T}:=$ $(-T) \vee u \wedge T$ belongs to $B V(\Omega)$ for every $T \geq 0$.

If $u \in G B V(\Omega)$, the function $\nabla u$ given by

$$
\nabla u=\nabla u^{T} \quad \mathcal{L}^{n} \text {-a.e. on }\{|u| \leq T\},
$$

turns out to be well-defined ([5], Th. 4.34). Moreover, the set function $T \mapsto S_{u^{T}}$ is monotone increasing; therefore, if we set ${ }^{1} S_{u}=\bigcup_{T>0} S_{u^{T}}$, by the Federer-Vol'pert Theorem, for $\mathcal{H}^{n-1}$-a.e. $x \in S_{u}$ we can consider the functions of $T$ :

$$
\left(u^{T}\right)^{-}(x), \quad\left(u^{T}\right)^{+}(x),
$$

which turn out to be monotone; then the traces:

$$
u^{-}(x)=\lim _{T \rightarrow+\infty}\left(u^{T}\right)^{-}(x), \quad u^{+}(x)=\lim _{T \rightarrow+\infty}\left(u^{T}\right)^{+}(x)
$$

are well-defined for $\mathcal{H}^{n-1}$-a.e. $x \in S_{u}$.

Finally, for a function $u \in G B V(\Omega)$, we define (see [5], Def. 4.33) $\left|D^{c} u\right|$ as the supremum (in the sense of measures) of $\left|D^{c} u^{T}\right|$ for $T>0$. It can be proved that for any Borel subset $B$ of $\Omega$

$$
\left|D^{c} u\right|(B)=\lim _{T \rightarrow+\infty}\left|D^{c} u^{T}\right|(B) .
$$

Slicing. Let us now recall some basic properties of one-dimensional sections of $B V$ functions; they will enter the so-called slicing methods to reduce to lower-dimensional statements (see, e.g., [4]). We first introduce some notation. Let $\xi \in S^{n-1}$, and let $\xi^{\perp}=\left\{y \in \mathbb{R}^{n}:\langle y, \xi\rangle=0\right\}$ be the linear hyperplane orthogonal to $\xi$. If $y \in \xi^{\perp}$ and $E \subseteq \mathbb{R}^{n}$ we set $E_{\xi, y}=\{t \in \mathbb{R}: y+t \xi \in E\}$. Moreover, for any given function $u: \Omega \rightarrow \mathbb{R}$ we define $u_{\xi, y}: \Omega_{\xi, y} \rightarrow \mathbb{R}$ by $u_{\xi, y}(t)=u(y+t \xi)$. For the results collected in the following theorem see [5], Section 3.11.

Theorem 2.3. Let $u \in B V(\Omega)$. Then $u_{\xi, y} \in B V\left(\Omega_{\xi, y}\right)$ for every $\xi \in S^{n-1}$ and for $\mathcal{H}^{n-1}$-a.e. $y \in \xi^{\perp}$. For such values of $y$ we have (denoting by $u_{\xi, y}^{\prime}$ the absolutely continuous part of the measure derivative of $u_{\xi, y}$ ):

$$
u_{\xi, y}^{\prime}(t)=\langle\nabla u(y+t \xi), \xi\rangle \text { for a.e. } t \in \Omega_{\xi, y} ; \quad S_{u_{\xi, y}}=\left(S_{u}\right)_{\xi, y} .
$$

Moreover, for every open subset $A$ of $\Omega$ we have

$$
\int_{\xi^{\perp}}\left|D^{c} u_{\xi, y}\right|\left(A_{\xi, y}\right) \mathrm{d} \mathcal{H}^{n-1}(y)=\left|\left\langle D^{c} u, \xi\right\rangle\right|(A),
$$

and for every positive Borel function $g$

$$
\int_{\xi^{\perp}} \sum_{t \in S_{u_{\xi}, y}} g(y+t \xi) \mathrm{d} \mathcal{H}^{n-1}(y)=\int_{S_{u}} g(x)\left|\left\langle\nu_{u}, \xi\right\rangle\right| \mathrm{d} \mathcal{H}^{n-1} .
$$

\footnotetext{
${ }^{1}$ It turns out that if $x \in \Omega \backslash S_{u}$ then $u$ has an approximate limit (in the sense of Federer) at $x$, i.e. there exists $z \in \mathbb{R}$ such that for any $\varepsilon>0$ the set $\{y \in \Omega:|u(y)-z|>\varepsilon\}$ has density 0 at $x$.
} 
Conversely, if $u \in L^{1}(\Omega)$ and for all $\xi \in\left\{e_{1}, \ldots, e_{n}\right\}$ and for a.e. $y \in \xi^{\perp}, u_{\xi, y} \in B V\left(\Omega_{\xi, y}\right)$ and

$$
\int_{\xi^{\perp}}\left|D u_{\xi, y}\right|\left(\Omega_{\xi, y}\right) \mathrm{d} \mathcal{H}^{n-1}(y)<+\infty
$$

then $u \in B V(\Omega)$.

Relaxation. We recall that the relaxed functional $\bar{F}$ of a given functional $F$ is the largest lower semicontinuous functional smaller than $F$. We will need the following relaxation theorem, which can be obtained from the results contained in [8] (see, in particular, the proof of Theorem 3.1 and use the case $f(t)=t+t^{2}$, too); see also [2], Theorem 3.2. Here $S B V^{2}(\Omega)=\left\{u \in S B V(\Omega):|\nabla u| \in L^{2}(\Omega), \mathcal{H}^{n-1}\left(S_{u}\right)<+\infty\right\}$.

Theorem 2.4. Let $\vartheta: \mathbb{R} \rightarrow[0,+\infty)$ be a continuous, concave function with $\vartheta(0)=0$ and $\lim _{t \rightarrow 0} \vartheta(t) / t=1$. Let $\Omega$ be a bounded open subset of $\mathbb{R}^{n}$, and consider the functional $F: B V(\Omega) \rightarrow[0,+\infty]$ defined by

$$
F(u)= \begin{cases}\int_{\Omega}|\nabla u(x)| \mathrm{d} x+\int_{S_{u}} \vartheta\left(\left|u^{+}(x)-u^{-}(x)\right|\right) \mathrm{d} \mathcal{H}^{n-1} & \text { if } u \in S B V^{2}(\Omega) \cap L^{\infty}(\Omega), \\ +\infty & \text { otherwise. }\end{cases}
$$

Then the relaxed functional $\bar{F}$ of $F$ on $B V(\Omega)$ with respect to the $L^{1}$-topology is given by

$$
\bar{F}(u)=\int_{\Omega}|\nabla u(x)| \mathrm{d} x+\int_{S_{u}} \vartheta\left(\left|u^{+}(x)-u^{-}(x)\right|\right) \mathrm{d} \mathcal{H}^{n-1}+\left|D^{c} u\right|(\Omega) .
$$

$\Gamma$-convergence. For the general theory see [19]. Let $(X, d)$ be a metric space. Let $\left(F_{j}\right)_{j \in \mathbb{N}}$ be a sequence of functions $X \rightarrow \overline{\mathbb{R}}$. We say that $\left(F_{j}\right) \Gamma$-converges, as $j \rightarrow+\infty$, to $F: X \rightarrow \overline{\mathbb{R}}$, if for all $u \in X$ we have:

i) (lower semicontinuity inequality) for every sequence $\left(u_{j}\right)$ converging to $u$

$$
F(u) \leq \liminf _{j \rightarrow+\infty} F_{j}\left(u_{j}\right)
$$

ii) (existence of a recovery sequence) there exists a sequence $\left(u_{j}\right)$ converging to $u$ such that:

$$
F(u) \geq \limsup _{j \rightarrow+\infty} F_{j}\left(u_{j}\right) .
$$

If $F_{j}$ equals the same functional $G$ for every $j$, then the $\Gamma$-limit is nothing but the relaxed functional of $G$.

The lower and upper $\Gamma$-limits of $\left(F_{j}\right)$ in $u \in X$ are defined as

$$
\begin{aligned}
& F^{\prime}(u)=\inf \left\{\liminf _{j \rightarrow+\infty} F_{j}\left(u_{j}\right): u_{j} \rightarrow u\right\}, \\
& F^{\prime \prime}(u)=\inf \left\{\limsup _{j \rightarrow+\infty} F_{j}\left(u_{j}\right): u_{j} \rightarrow u\right\},
\end{aligned}
$$

respectively.

We extend this definition of convergence to families depending on a real parameter. Given a family $\left(F_{\varepsilon}\right)_{\varepsilon>0}$ of functions $X \rightarrow \overline{\mathbb{R}}$, we say that it $\Gamma$-converges, as $\varepsilon \rightarrow 0$, to $F: X \rightarrow \overline{\mathbb{R}}$ if for every positive infinitesimal sequence $\left(\varepsilon_{j}\right)$ the sequence $\left(F_{\varepsilon_{j}}\right) \Gamma$-converges to $F$.

If we define the lower and upper $\Gamma$-limits of $\left(F_{\varepsilon}\right)$ as

$$
\begin{aligned}
F^{\prime}(u) & =\inf \left\{\liminf _{\varepsilon \rightarrow 0} F_{\varepsilon}\left(u_{\varepsilon}\right): u_{\varepsilon} \rightarrow u\right\}, \\
F^{\prime \prime}(u) & =\inf \left\{\limsup _{\varepsilon \rightarrow 0} F_{\varepsilon}\left(u_{\varepsilon}\right): u_{\varepsilon} \rightarrow u\right\},
\end{aligned}
$$


respectively, then $\left(F_{\varepsilon}\right) \Gamma$-converges to $F$ in $u$ if and only if $F^{\prime}(u)=F^{\prime \prime}(u)=F(u)$. Both $F^{\prime}$ and $F^{\prime \prime}$ are lower semicontinuous on $X$. In the estimate of $F^{\prime}$ we shall use the following immediate consequence of the definition:

$$
F^{\prime}(u)=\inf \left\{\liminf _{j \rightarrow+\infty} F_{\varepsilon_{j}}\left(u_{j}\right): \varepsilon_{j} \rightarrow 0^{+}, u_{j} \rightarrow u\right\} .
$$

It turns out that the infimum is attained.

We will also use the fact (see [19], Prop. 6.11) that the lower and upper $\Gamma$-limits of a sequence of functionals coincide with the lower and upper $\Gamma$-limits, respectively, of the sequence of the corresponding relaxed functionals.

An important consequence of the definition of $\Gamma$-convergence is the following result about the convergence of minimizers (see, e.g., [19], Cor. 7.20):

Theorem 2.5. Let $F_{j}: X \rightarrow \overline{\mathbb{R}}(j \in \mathbb{N})$ be a sequence of functions which $\Gamma$-converge to some $F: X \rightarrow \overline{\mathbb{R}}$; assume that $\inf _{v \in X} F_{j}(v)>-\infty$ for every $j$. Let $\left(\varepsilon_{j}\right)$ be a positive infinitesimal sequence, and for every $j$ let $u_{j} \in X$ be an $\varepsilon_{j}$-minimizer of $F_{j}$, i.e.

$$
F_{j}\left(u_{j}\right) \leq \inf _{v \in X} F_{j}(v)+\varepsilon_{j} .
$$

Assume that $u_{j} \rightarrow u$ for some $u \in X$. Then $u$ is a minimum point of $F$, and $F(u)=\lim _{j \rightarrow+\infty} F_{j}\left(u_{j}\right)$.

Remark 2.6. We also point out the following property, which is a direct consequence of the definition of $\Gamma$-convergence: if $F_{\varepsilon} \stackrel{\Gamma}{\rightarrow} F$ then $F_{\varepsilon}+G \stackrel{\Gamma}{\rightarrow} F+G$ whenever $G$ is continuous.

In conclusion we recall the following useful tool, which can be found in [10].

Lemma 2.7 (supremum of measures). Let $\Omega$ be an open subset of $\mathbb{R}^{n}$ and denote by $\mathcal{A}(\Omega)$ the family of its open subsets. Let $\lambda$ be a positive Borel measure on $\Omega$, and $\mu: \mathcal{A}(\Omega) \rightarrow[0,+\infty)$ a set function which is superadditive on open sets with disjoint compact closures (i.e. if $A, B \subset \subset \Omega$ and $\bar{A} \cap \bar{B}=\emptyset$, then $\mu(A \cup B) \geq \mu(A)+\mu(B)$ ). Let $\left(\psi_{i}\right)_{i \in I}$ be a family of positive Borel functions. Suppose that

$$
\mu(A) \geq \int_{A} \psi_{i} \mathrm{~d} \lambda \quad \text { for every } A \in \mathcal{A}(\Omega) \text { and } i \in I ;
$$

then

$$
\mu(A) \geq \int_{A} \sup _{i} \psi_{i} \mathrm{~d} \lambda \quad \text { for every } A \in \mathcal{A}(\Omega) .
$$

Corollary 2.8. Let $\mu$ be as in Lemma 2.7. Let $\lambda_{1}, \lambda_{2}$ be mutually singular Borel measures, and $\psi_{1}, \psi_{2}$ positive Borel functions. Assume that

$$
\mu(A) \geq \int_{A} \psi_{i} \mathrm{~d} \lambda_{i} \quad \text { for every } A \in \mathcal{A}(\Omega) \text { and } i=1,2 .
$$

Then

$$
\mu(A) \geq \int_{A} \psi_{1} \mathrm{~d} \lambda_{1}+\int_{A} \psi_{2} \mathrm{~d} \lambda_{2} \quad \text { for every } A \in \mathcal{A}(\Omega) .
$$

Proof. Let $E \subseteq \Omega$ be such that $\lambda_{1}(\Omega \backslash E)=0$ and $\lambda_{2}(E)=0$. Then we can suppose that $\psi_{1}=0$ on $\Omega \backslash E$ and $\psi_{2}=0$ on $E$. Then $\psi_{1} \vee \psi_{2}=\psi_{1}+\psi_{2}$. We now conclude by applying the preceeding lemma with $\lambda=\lambda_{1}+\lambda_{2}$. 


\section{Statement of the Results}

Let $\Omega \subseteq \mathbb{R}^{n}$ be a bounded open set with Lipschitz boundary, and $f:[0,+\infty) \rightarrow[0,+\infty)$ be a non-decreasing, strictly concave and $C^{2}$ function such that

$$
\lim _{t \rightarrow 0^{+}} \frac{f(t)}{t}=1 .
$$

For every $\varepsilon>0$ let $F_{\varepsilon}: L^{1}(\Omega) \rightarrow \mathbb{R}$, be defined by

$$
F_{\varepsilon}(u)= \begin{cases}\frac{1}{\varepsilon} \int_{\Omega} f\left(\varepsilon f_{B_{\varepsilon}(x) \cap \Omega}|\nabla u(y)| \mathrm{d} y\right) \mathrm{d} x & \text { if } u \in W^{1,1}(\Omega) \\ +\infty & \text { otherwise. }\end{cases}
$$

The main result is the following theorem:

Theorem 3.1. The family $\left(F_{\varepsilon}\right)_{\varepsilon>0}$, defined in (3.1), $\Gamma$-converges, in the $L^{1}$-topology as $\varepsilon \rightarrow 0$, to the functional $\mathcal{F}: L^{1}(\Omega) \rightarrow[0,+\infty]$ given by

$$
\mathcal{F}(u)= \begin{cases}\int_{\Omega}|\nabla u(x)| \mathrm{d} x+\int_{S_{u}} \vartheta\left(\left|u^{+}(x)-u^{-}(x)\right|\right) \mathrm{d} \mathcal{H}^{n-1}+\left|D^{c} u\right|(\Omega) & \text { if } u \in G B V(\Omega) \\ +\infty & \text { otherwise }\end{cases}
$$

with

$$
\vartheta(s)=2 \int_{0}^{1} f\left(\frac{\omega_{n-1}}{\omega_{n}} s\left(\sqrt{1-t^{2}}\right)^{n-1}\right) \mathrm{d} t \quad(s>0)
$$

where $\omega_{k}$ denotes the volume of the $k$-dimensional ball in $\mathbb{R}^{k}$ (with $\omega_{0}=1$ ).

If $n=1$ and $\Omega=(a, b)$, this result follows as a particular case of Theorem 3.3 in [21]; the corresponding form of the jump energy density is

$$
\vartheta(s)=2 f\left(\frac{s}{2}\right) .
$$

Theorem 3.2 (compactness). Let $\left(\varepsilon_{j}\right)$ be a positive infinitesimal sequence, and let $\left(u_{j}\right)$ be a sequence in $L^{1}(\Omega)$ such that $\left\|u_{j}\right\|_{\infty} \leq M$, and $F_{\varepsilon_{j}}\left(u_{j}\right) \leq M$ for a suitable constant $M$ independent of $j$. Then there exists a subsequence $\left(u_{j_{k}}\right)$ converging in $L^{1}(\Omega)$ to a function $u \in B V(\Omega)$.

As an example of application of these results we consider the following corollary.

Corollary 3.3. Let $\left(\varepsilon_{j}\right)$ be a positive infinitesimal sequence and $g \in L^{\infty}(\Omega)$. For every $u \in L^{1}(\Omega)$ and $j \in \mathbb{N}$, define:

$$
G_{j}(u)=F_{\varepsilon_{j}}(u)+\int_{\Omega}|u(x)-g(x)| \mathrm{d} x,
$$

and

$$
\mathcal{G}(u)=\mathcal{F}(u)+\int_{\Omega}|u(x)-g(x)| \mathrm{d} x .
$$

For every $j$ let $u_{j}$ be a $\sigma_{j}$-minimizer of $G_{j}$ in $L^{1}(\Omega)$ (with $\sigma_{j} \searrow 0$ ), i.e. $G_{j}\left(u_{j}\right) \leq \inf _{L^{1}(\Omega)} G_{j}+\sigma_{j}$. Then $\left(u_{j}\right)$ converges, up to a subsequence, to a minimizer of $\mathcal{G}$ in $L^{1}(\Omega)$.

Proof. Since $g \in L^{\infty}(\Omega)$ and $F_{\varepsilon_{j}}$ decreases by truncation, we can assume that $\left(u_{j}\right)$ is equibounded. By Theorem 3.2 there exists $u \in B V(\Omega)$ such that (up to a subsequence) $u_{j} \rightarrow u$ in $L^{1}(\Omega)$. By Theorem 2.5, since $\left(G_{j}\right) \Gamma$-converge to $\mathcal{G}$ (recall Rem. 2.6), $u$ is a minimum point of $\mathcal{G}$ on $L^{1}(\Omega)$. 
Remark 3.4. We will need the following "localization" of the functional $F_{\varepsilon}$ : for every open subset $A$ of $\Omega$, we set

$$
F_{\varepsilon}(u, A)= \begin{cases}\frac{1}{\varepsilon} \int_{A} f\left(\varepsilon f_{B_{\varepsilon}(x) \cap \Omega}|\nabla u(y)| \mathrm{d} y\right) \mathrm{d} x & \text { if } u \in W^{1,1}(\Omega) \\ +\infty & \text { if } u \in L^{1}(\Omega) \backslash W^{1,1}(\Omega) .\end{cases}
$$

Clearly, $F_{\varepsilon}(\cdot, \Omega)$ coincides with the functional $F_{\varepsilon}$ defined in $(3.1)$. The lower and upper $\Gamma$-limits of $\left(F_{\varepsilon}(\cdot, A)\right)$ (see Sect. 2) will be denoted by $F^{\prime}(\cdot, A)$ and $F^{\prime \prime}(\cdot, A)$, respectively.

Note that, if $A \subset \subset \Omega$, the lower and upper $\Gamma$-limits of $\left(F_{\varepsilon}(\cdot, A)\right)$ do not change by replacing $\Omega$ with any $\Omega^{\prime} \supset$ つ.

Remark 3.5. The one-dimensional version of Theorem 3.1 will be used in the volume estimate of the lower $\Gamma$-limit (see Prop. 4.7) when applying the so-called slicing method. Actually, we will need the stronger form of the lower $\Gamma$-limit estimate contained in Remark 3.4 of [21]: if $n=1$ and $\Omega=(a, b)$, then

$$
F^{\prime}(u, A) \geq \int_{A}\left|u^{\prime}(x)\right| \mathrm{d} x+2 \sum_{x \in S_{u} \cap A} f\left(\frac{1}{2}\left|u^{+}(x)-u^{-}(x)\right|\right)+\left|D^{c} u\right|(A)
$$

for every $u \in G B V(\Omega)$ and $A$ open subset of $\Omega$. The extension to an arbitrary bounded open subset $\Omega$ of $\mathbb{R}$ and $A \subset \subset \Omega$ is immediate.

In the computation of the upper $\Gamma$-limit we will use the following result. Here the Lipschitz regularity of $\partial \Omega$ guarantees that a function $u \in B V(\Omega)$ admits a $B V$-extension on a neighborhood of $\Omega$ (see, e.g., [5], Prop. 3.21); and that there exists a constant $\gamma>0$ such that:

$$
\left|B_{\varepsilon}(x) \cap \Omega\right| \geq \gamma \varepsilon^{n}
$$

for any $x \in \Omega$ and $\varepsilon<\operatorname{diam}(\Omega)$.

Proposition 3.6. For every $\varepsilon>0$, the relaxed functional of $F_{\varepsilon}$ in the $L^{1}$-topology is given by

$$
\bar{F}_{\varepsilon}(u)=\frac{1}{\varepsilon} \int_{\Omega} f\left(\frac{\varepsilon}{\left|B_{\varepsilon}(x) \cap \Omega\right|}|D u|\left(B_{\varepsilon}(x) \cap \Omega\right)\right) \mathrm{d} x
$$

for every $u \in B V(\Omega)$.

Proof. Denote by $H_{\varepsilon}$ the functional on the right-hand side of (3.4), defined with value $+\infty$ on $L^{1} \backslash B V$. Let $c_{\varepsilon}(x)=\varepsilon /\left|B_{\varepsilon}(x) \cap \Omega\right|$. It is easy to prove the $L^{1}$-lower semicontinuity of $H_{\varepsilon}$ at every $u \in B V$. Indeed: let $\left(u_{h}\right)$ be a sequence in $L^{1}(\Omega)$ converging to a function $u \in B V(\Omega)$; we can suppose that $\liminf _{h \rightarrow+\infty} H_{\varepsilon}\left(u_{h}\right)$ is finite and is a limit, and that each $u_{h}$ is in $B V$; then by Fatou's Lemma, the monotonicity of $f$ and the lower semicontinuity of the total variation, we have:

$$
\begin{aligned}
\liminf _{h \rightarrow+\infty} & H_{\varepsilon}\left(u_{h}\right) \geq \frac{1}{\varepsilon} \int_{\Omega} \liminf _{h \rightarrow+\infty} f\left(c_{\varepsilon}(x)\left|D u_{h}\right|\left(B_{\varepsilon}(x) \cap \Omega\right)\right) \mathrm{d} x \\
& \geq \frac{1}{\varepsilon} \int_{\Omega} f\left(c_{\varepsilon}(x) \liminf _{h \rightarrow+\infty}\left|D u_{h}\right|\left(B_{\varepsilon}(x) \cap \Omega\right)\right) \mathrm{d} x \\
& \geq \frac{1}{\varepsilon} \int_{\Omega} f\left(c_{\varepsilon}(x)|D u|\left(B_{\varepsilon}(x) \cap \Omega\right)\right) \mathrm{d} x=H_{\varepsilon}(u) .
\end{aligned}
$$


Since $H_{\varepsilon}(u) \leq F_{\varepsilon}(u)$ for all $u \in L^{1}(\Omega)$, the relaxed functional $\bar{F}_{\varepsilon}$ is estimated from below by $H_{\varepsilon}$ on $B V(\Omega)$. Consider now the opposite inequality. Given $u \in B V(\Omega)$, if $\left(v_{h}\right)$ denotes the sequence obtained from $u$ (extended to a neighborhood of $\Omega$ ) by standard mollification, then $v_{h} \rightarrow u$ in $L^{1}(\Omega)$ and

$$
\left.\left|D v_{h}\right|\left(B_{\varepsilon}(x) \cap \Omega\right)\right) \rightarrow|D u|\left(B_{\varepsilon}(x) \cap \Omega\right)
$$

for a.e. $x \in \Omega$ (see, e.g., [5] Prop. 3.7 and Rem. 3.8: note that $D^{c} u$ vanishes on the sets with finite $\mathcal{H}^{n-1}$ measure; moreover, if $S$ is $\sigma$-finite with respect to $\mathcal{H}^{n-1}$, then $\left\{x \in \Omega: \mathcal{H}^{n-1}\left(S \cap \partial B_{\varepsilon}(x)\right)>0\right\}$ is at most countable). Then by the dominated convergence Theorem (recall (3.3) and that $f(t) \leq t)$ :

$$
\lim _{h \rightarrow+\infty} F_{\varepsilon}\left(v_{h}\right)=\frac{1}{\varepsilon} \int_{\Omega} f\left(c_{\varepsilon}(x)|D u|\left(B_{\varepsilon}(x) \cap \Omega\right)\right) \mathrm{d} x=H_{\varepsilon}(u) .
$$

This shows that $H_{\varepsilon}$ coincides with the relaxed functional $\bar{F}_{\varepsilon}$ on $B V(\Omega)$.

Remark 3.7. From the preceeding proposition we easily deduce that $F^{\prime \prime}(u)<+\infty$ if $u \in B V(\Omega)$. Indeed, the upper $\Gamma$-limit of $\left(F_{\varepsilon}\right)$ coincides with the upper $\Gamma$-limit of $\left(\bar{F}_{\varepsilon}\right)$; therefore, since $f(t) \leq t$, if we set $\mu=|D u|$, we have:

$$
F^{\prime \prime}(u) \leq \limsup _{\varepsilon \rightarrow 0} \bar{F}_{\varepsilon}(u) \leq \limsup _{\varepsilon \rightarrow 0} \int_{\Omega} \frac{1}{\left|B_{\varepsilon}(x) \cap \Omega\right|} \int_{\Omega} 1_{B_{\varepsilon}(x)} \mathrm{d} \mu \mathrm{d} x,
$$

where $1_{B_{\varepsilon}(x)}$ is the characteristic function of $B_{\varepsilon}(x)$; therefore, by (3.3):

$$
F^{\prime \prime}(u) \leq \limsup _{\varepsilon \rightarrow 0} \frac{1}{\gamma \varepsilon^{n}} \int_{\Omega} \mathrm{d} \mu(y) \int_{\Omega} 1_{B_{\varepsilon}(x)}(y) \mathrm{d} x \leq \frac{\omega_{n}}{\gamma} \mu(\Omega)<+\infty .
$$

\section{Compactness. Lower BOUnd in terms of the volume and Cantor parts}

In [12] a crucial point in the proof of a lower bound for the $\Gamma$-limit of $\left(F_{\varepsilon}\right)$ in terms of the volume part of the Mumford-Shah functional is the estimate from below of $F_{\varepsilon}(u)$ through $(1-\delta) \int_{\Omega}|\nabla v|^{2} \mathrm{~d} x$, where $v$ is a function close to $u$ in $L^{1}$ and $\delta$ is arbitrary in $(0,1)$ (see [12], Prop. 4.1). The presence of the $L^{2}$-norm of the gradient yields, via the $S B V$ compactness theorem, separate semicontinuity inequalities for the absolutely continuous and the singular parts of the derivatives, thus giving the required bound in terms of the volume part. The proof of the cited proposition relies on a delicate partitioning of $\mathbb{R}^{n}$ by means of coordinate squares which are well-behaved with respect to the balls over which the gradients are averaged. In a $L^{1}$-context a perfectly analogous result, contained in Lemma 4.1 below, can thus be obtained with essentially the same proof, but now this does not imply the needed lower semicontinuity inequality for the volume parts. However, from Lemma 4.1 the compactness property of Theorem 3.2 for $\left(F_{\varepsilon}\right)$ easily follows.

The proof of the correct lower bound for the $\Gamma$-limit (see Prop. 4.7) will be obtained by a slicing technique. The main difficulty is that $F_{\varepsilon}(u)$, due to its non-local character, can not be simply expressed through the onedimensional sections of $u$. Thus, for any direction $\xi \in S^{n-1}$ we estimate $F_{\varepsilon}(u)$ through a suitable functional where the average on balls is replaced by the average on squares with a face orthogonal to $\xi$ (Lem. 4.3); this allows to split the average itself into a part along $\xi$ and another in the orthogonal space $\xi^{\perp}$. A slicing method can now be applied: the one-dimensional sections of $u$ are replaced by its averages along $\xi^{\perp}$ (see Lem. 4.4).

For every open subset $A$ of $\Omega$ and $\varrho>0$ we set:

$$
A_{\varrho}=\{x \in A: d(x, \partial A)>\varrho\} .
$$


Lemma 4.1. Let $A$ be an open subset of $\Omega$, and let $u \in W^{1,1}(\Omega) \cap L^{\infty}(\Omega)$. For every $\varepsilon>0$ and $\delta>0$, there exists a function $v \in S B V(A) \cap L^{\infty}(A)$ such that:

$$
\begin{aligned}
& (1-\delta) \int_{A}|\nabla v(x)| \mathrm{d} x \leq F_{\varepsilon}(u, A), \quad \mathcal{H}^{n-1}\left(S_{v} \cap A_{6 \varepsilon}\right) \leq c F_{\varepsilon}(u, A), \\
& \|v\|_{L^{\infty}(A)} \leq\|u\|_{L^{\infty}(A)}, \quad\|v-u\|_{L^{1}\left(A_{6 \varepsilon}\right)} \leq c \varepsilon F_{\varepsilon}(u, A)\|u\|_{L^{\infty}(A)},
\end{aligned}
$$

where $c$ is a constant depending only on $n, \delta$ and $f$.

Proof of Theorem 3.2. Let $A \subset \subset \Omega$, with $\partial A$ smooth. By Lemma 4.1 there exists a sequence $\left(v_{j}\right)$ in $S B V(A)$ and a constant $C$ independent of $A$ such that $\left\|v_{j}\right\|_{B V(A)} \leq C$ and $\left\|v_{j}\right\|_{L^{\infty}(A)} \leq M$; moreover, $\left\|v_{j}-u_{j}\right\|_{L^{1}(A)} \rightarrow 0$. Therefore, there exists a subsequence $\left(v_{j_{k}}\right)$ which converges to a function $u \in B V(A)$, with $\|u\|_{B V(A)} \leq C$. Hence $u \in B V(\Omega)$, too. Clearly, also $\left(u_{j_{k}}\right)$ converges to $u$ in $L^{1}(A)$. The arbitrariness of $A$ and a diagonal argument allow to find a subsequence $\left(u_{j_{k}}\right)$ which converges in $L_{\mathrm{loc}}^{1}(\Omega)$ to a function $u \in B V_{\mathrm{loc}}(\Omega)$; the uniform bound of $\left\|u_{j}\right\|_{L^{\infty}(\Omega)}$ implies the $L^{1}(\Omega)$-convergence of $\left(u_{j_{k}}\right)$ to $u$.

Corollary 4.2. Let $\left(\varepsilon_{j}\right)$ be a positive infinitesimal sequence and let $\left(u_{j}\right)$ be sequence in $L^{1}(\Omega)$ which converges to a function $u$. If $\left(F_{\varepsilon_{j}}\left(u_{j}\right)\right)$ is bounded, then $u \in G B V(\Omega)$. In particular, if $F^{\prime}(u)<+\infty$ then $u \in G B V(\Omega)$.

Proof. For each $T>0$ apply Theorem 3.2 to $u_{j}^{T}=(-T) \vee u_{j} \wedge T$ : we get $(-T) \vee u \wedge T \in B V(\Omega)$; hence $u \in G B V(\Omega)$.

Let $\xi \in S^{n-1}$ and let $\nu_{1}, \ldots, \nu_{n}$ be an orthonormal basis of $\mathbb{R}^{n}$, with $\nu_{1}=\xi$. For every $r>0$ and $x \in \mathbb{R}^{n}$, define:

$$
Q_{r}^{\xi}(x)=\left\{y \in \mathbb{R}^{n}:\left|\left\langle y-x, \nu_{i}\right\rangle\right|<r, \quad i=1, \ldots, n\right\} .
$$

Lemma 4.3. There exists a sequence $\left(c_{h}\right)_{h \in \mathbb{N}}$ of positive real numbers, with $c_{h} \stackrel{h}{\rightarrow} 1$, such that, for every $u \in W^{1,1}(\Omega), \xi \in \mathbb{R}^{n}$ and $A, A^{\prime}$ open subsets of $\Omega$, with $A^{\prime} \subset \subset A$, and for every $\varepsilon<d\left(A^{\prime}, \partial A\right) / 2$, the following inequality holds:

$$
F_{\varepsilon}(u, A) \geq \frac{1}{h \sigma_{\varepsilon}^{h}} \int_{A^{\prime}} f^{h}\left(\sigma_{\varepsilon}^{h} f_{Q_{\sigma_{\varepsilon}^{h}}^{\xi}(z)}|\nabla u(y)| \mathrm{d} y\right) \mathrm{d} z,
$$

where $f^{h}(t)=f\left(c_{h} h t\right)$ and $\sigma_{\varepsilon}^{h}=\varepsilon / h$.

Proof. For ease of notation we drop the superscript $\xi$ in $Q_{r}^{\xi}(z)\left(\xi \in S^{n-1}\right.$ is fixed). For every $h \in \mathbb{N}$ let

$$
Z_{h}=\left\{\alpha \in \mathbb{Z}^{n}: Q_{1 / h}(2 \alpha / h) \subseteq B_{1}(0)\right\}, \quad N_{h}=\# Z_{h} .
$$

Clearly

and, for every $\varepsilon>0$ and $x \in \mathbb{R}^{n}$

$$
c_{h}:=\frac{2^{n} N_{h}}{\omega_{n} h^{n}} \stackrel{h}{\rightarrow} 1
$$

$$
Z_{h}=\left\{\alpha \in \mathbb{Z}^{n}: Q_{\varepsilon}^{h}(x, \alpha):=x+Q_{\varepsilon / h}(2 \alpha \varepsilon / h) \subseteq B_{\varepsilon}(x)\right\} .
$$

Fix now $u \in W^{1,1}(\Omega)$; then for every $\varepsilon>0$ and $x \in \Omega$, with $d(x, \partial \Omega)>\varepsilon$ :

$$
f_{B_{\varepsilon}(x)}|\nabla u(y)| \mathrm{d} y \geq \sum_{\alpha \in Z_{h}} \frac{\left(\frac{2 \varepsilon}{h}\right)^{n}}{\omega_{n} \varepsilon^{n}} f_{Q_{\varepsilon}^{h}(x, \alpha)}|\nabla u(y)| \mathrm{d} y=\sum_{\alpha \in Z_{h}} \frac{c_{h}}{N_{h}} f_{Q_{\varepsilon}^{h}(x, \alpha)}|\nabla u(y)| \mathrm{d} y .
$$


By the concavity of $f$ :

$$
f\left(\varepsilon f_{B_{\varepsilon}(x)}|\nabla u(y)| \mathrm{d} y\right) \geq \sum_{\alpha \in Z_{h}} \frac{1}{N_{h}} f\left(\varepsilon c_{h} f_{Q_{\varepsilon}^{h}(x, \alpha)}|\nabla u(y)| \mathrm{d} y\right)
$$

Let $A^{\prime} \subset \subset A$, and $2 \varepsilon<d\left(A^{\prime}, \partial A\right)$; we can find an open subset $A^{\prime \prime}$ of $A$ such that

$$
A^{\prime} \subset \subset A^{\prime \prime} \subset \subset A, \quad d\left(A^{\prime}, \partial A^{\prime \prime}\right)>\varepsilon, \quad d\left(A^{\prime \prime}, \partial A\right)>\varepsilon
$$

Thus, if we set $\sigma_{\varepsilon}^{h}=\varepsilon / h$, we get:

$$
F_{\varepsilon}\left(u, A^{\prime \prime}\right) \geq \sum_{\alpha \in Z_{h}} \frac{1}{N_{h}}\left(\frac{1}{h \sigma_{\varepsilon}^{h}} \int_{A^{\prime \prime}} f^{h}\left(\sigma_{\varepsilon}^{h} f_{Q_{\varepsilon}^{h}(x, \alpha)}|\nabla u(y)| \mathrm{d} y\right) \mathrm{d} x\right)
$$

with $f^{h}$ as in the statement of the lemma. The change of variables $z=x+2 \sigma_{\varepsilon}^{h} \alpha$ now yields:

$$
F_{\varepsilon}(u, A) \geq \sum_{\alpha \in Z_{h}} \frac{1}{N_{h}}\left(\frac{1}{h \sigma_{\varepsilon}^{h}} \int_{A^{\prime}} f^{h}\left(\sigma_{\varepsilon}^{h} f_{Q_{\sigma_{\varepsilon}^{h}}(z)}|\nabla u(y)| \mathrm{d} y\right) \mathrm{d} z\right) .
$$

Since the $N_{h}$ terms of the sum do not depend on $\alpha$, we conclude.

Given $y \in \mathbb{R}^{n-1}$ and $r>0$, define

$$
\tilde{Q}_{r}(y)=\left\{z \in \mathbb{R}^{n-1}:\left|z_{i}-y_{i}\right|<r, i=1, \ldots, n-1\right\}
$$

Lemma 4.4. Let $\left(\varepsilon_{j}\right)$ be a positive infinitesimal sequence. Let $A$ be an open subset of $\mathbb{R}^{n-1}$, and $a, b \in \mathbb{R}$, with $a<b$. Let $u_{j}, u \in L^{1}((a, b) \times A)(j \in \mathbb{N})$ and $u_{j} \rightarrow u$ in $L^{1}((a, b) \times A)$. For a.e. $t \in(a, b)$, for every $y \in A$ and $j \in \mathbb{N}$ with $\varepsilon_{j}<\frac{1}{\sqrt{n-1}} d(y, \partial A)$ we can define

$$
v_{j}(t, y)=f_{\tilde{Q}_{\varepsilon_{j}}(y)} u_{j}(t, z) \mathrm{d} z
$$

Then there exists a subsequence $\left(v_{j_{k}}\right)_{k}$ such that $v_{j_{k}}(\cdot, y) \rightarrow u(\cdot, y)$ in $L^{1}(a, b)$ for a.e. $y \in A$.

Proof. There exists $N \subseteq(a, b)$, with $|N|=0$ such that $u_{j}(t, \cdot), u(t, \cdot) \in L^{1}(A)$ for every $t \in(a, b) \backslash N$. In particular, $v_{j}(t, y)$ is well-defined for every $t \in(a, b) \backslash N, y \in A$, and $\varepsilon_{j}<d(y, \partial A) / \sqrt{n-1}$. Let

$$
\phi_{j}(z)=\frac{1}{\left|\tilde{Q}_{\varepsilon_{j}}(0)\right|} \chi_{\tilde{Q}_{\varepsilon_{j}}(0)}(z)
$$

where $\chi_{\tilde{Q}_{\varepsilon_{j}}(0)}$ denotes the characteristic function of $\tilde{Q}_{\varepsilon_{j}}(0)$. We have

$$
\begin{aligned}
& \int_{A}\left(\int_{a}^{b}\left|v_{j}(t, y)-u(t, y)\right| \mathrm{d} t\right) \mathrm{d} y=\int_{A} \mathrm{~d} y \int_{a}^{b}\left|\int_{\mathbb{R}^{n-1}} u_{j}(t, z) \phi_{j}(z-y) \mathrm{d} z-u(t, y)\right| \mathrm{d} t \\
& \quad \leq \int_{A} \mathrm{~d} y \int_{a}^{b} \mathrm{~d} t \int_{\mathbb{R}^{n-1}}\left|u_{j}(t, z)-u(t, z)\right| \phi_{j}(z-y) \mathrm{d} z+\int_{A} \mathrm{~d} y \int_{a}^{b}\left|\int_{\mathbb{R}^{n-1}} u(t, z) \phi_{j}(z-y) d z-u(t, y)\right| \mathrm{d} t .
\end{aligned}
$$


Denote these two integral terms by $I_{j}^{\prime}$ and $I_{j}^{\prime \prime}$ respectively. Then

$$
\begin{aligned}
I_{j}^{\prime \prime} \leq \int_{a}^{b} \mathrm{~d} t \int_{A} \mathrm{~d} y \int_{\mathbb{R}^{n-1}}|u(t, z)-u(t, y)| \phi_{j}(z-y) \mathrm{d} z=\int_{a}^{b} \mathrm{~d} t \int_{A} \mathrm{~d} y \int_{\mathbb{R}^{n-1}}|u(t, y+w)-u(t, y)| \phi_{j}(w) \mathrm{d} w \\
=\int_{\mathbb{R}^{n-1}} \phi_{j}(w)\|u(\cdot, \cdot+w)-u\|_{L^{1}((a, b) \times A)} \mathrm{d} w \leq \sup _{|w| \leq \varepsilon_{j} \sqrt{n-1}}\|u(\cdot, \cdot+w)-u\|_{L^{1}((a, b) \times A)} .
\end{aligned}
$$

Since translation is continuous in the $L^{1}$ norm, $I_{j}^{\prime \prime}$ tends to 0 as $j \rightarrow+\infty$. Let us now consider $I_{j}^{\prime}$;

$I_{j}^{\prime}=\int_{A}\left(\int_{a}^{b}\left(\left|u_{j}-u\right|(t, \cdot) * \phi_{j}\right)(y) \mathrm{d} t\right) \mathrm{d} y \leq \int_{a}^{b}\left\|\left(u_{j}-u\right)(t, \cdot)\right\|_{L^{1}(A)}\left\|\phi_{j}\right\|_{L^{1}\left(\mathbb{R}^{n-1}\right)} \mathrm{d} t=\left\|u_{j}-u\right\|_{L^{1}((a, b) \times A)}$,

which tends to 0 as $j \rightarrow+\infty$. Thus we conclude that for every $A$ open subset of $\mathbb{R}^{n-1}$

$$
\lim _{j \rightarrow+\infty} \int_{A}\left(\int_{a}^{b}\left|v_{j}(t, y)-u(t, y)\right| \mathrm{d} t\right) \mathrm{d} y=0 .
$$

In particular, there exists a subsequence $\left(v_{j_{k}}\right)_{k}$ such that $v_{j_{k}}(\cdot, y) \rightarrow u(\cdot, y)$ in $L^{1}(a, b)$ for a.e. $y \in A$.

Remark 4.5. The result of the previous lemma can be immediately generalized to the case where $(a, b)$ is replaced by any bounded open subset of $\mathbb{R}$.

Remark 4.6. Given $u \in L^{1}(a, b)$, the set functions $A \mapsto F_{\varepsilon}(u, A)$ are increasing and superadditive. Consequently, also the set function $A \mapsto F^{\prime}(u, A)$ is increasing and superadditive, i.e.

(i) $F^{\prime}\left(u, A_{1}\right) \leq F^{\prime}\left(u, A_{2}\right)$, whenever $A_{1} \subseteq A_{2} \subseteq \Omega$;

(ii) $F^{\prime}\left(u, A_{1} \cup A_{2}\right) \geq F^{\prime}\left(u, A_{1}\right)+F^{\prime}\left(u, A_{2}\right)$, whenever $A_{1} \cap A_{2}=\emptyset$.

Proposition 4.7. For every $u \in B V(\Omega)$ and $A \in \mathcal{A}(\Omega)$

$$
F^{\prime}(u, A) \geq \int_{A}|\nabla u(x)| \mathrm{d} x, \quad F^{\prime}(u, A) \geq\left|D^{c} u\right|(A) .
$$

Proof. Let $\left(\varepsilon_{j}\right)$ be a positive infinitesimal sequence and let $\left(u_{j}\right)$ be a sequence in $W^{1,1}(\Omega)$ converging to $u \in L^{1}(\Omega)$ and such that $F_{\varepsilon_{j}}\left(u_{j}, A\right) \rightarrow F^{\prime}(u, A)$ as $j \rightarrow+\infty$. Let $\xi \in S^{n-1}$. By Lemma 4.3, applied with $\varepsilon=\varepsilon_{j}$ and $u=u_{j}$, and by Fubini's Theorem, if $A$ and $A^{\prime}$ are open subsets of $\Omega$, with $A^{\prime} \subset \subset A$, and $2 \varepsilon_{j}<d\left(A^{\prime}, \partial A\right)$, we have (here $\left.\sigma_{j}^{h}=\varepsilon_{j} / h\right)$ :

$$
\begin{aligned}
F_{\varepsilon_{j}}\left(u_{j}, A\right) \geq \frac{1}{h \sigma_{j}^{h}} \int_{A^{\prime}} f^{h}\left(\sigma_{j}^{h} f_{Q_{\sigma_{j}^{h}}^{\xi}(z)}\left|\nabla u_{j}(x)\right| \mathrm{d} x\right) \mathrm{d} z & \\
& =\int_{\xi^{\perp}}\left(\frac{1}{h \sigma_{j}^{h}} \int_{A_{\xi, y}^{\prime}} f^{h}\left(\sigma_{j}^{h} f_{Q_{\sigma_{j}^{h}}^{\xi}(y+t \xi)}\left|\nabla u_{j}(x)\right| \mathrm{d} x\right) \mathrm{d} t\right) \mathrm{d} \mathcal{H}^{n-1}(y)
\end{aligned}
$$

where $\xi^{\perp}$ and $A_{\xi, y}^{\prime}$ stand for the subspace orthogonal to $\xi$ and for the one-dimensional section of $A^{\prime}$ in the direction $\xi$, as in Theorem 2.3. It is not restrictive to assume $\xi=e_{1}$ (and we will drop the superscript $\xi$ ). Then 
we can rewrite:

$$
F_{\varepsilon_{j}}\left(u_{j}, A\right) \geq \int_{\tilde{A}^{\prime}}\left(\frac{1}{h \sigma_{j}^{h}} \int_{A_{y}^{\prime}} f^{h}\left(\sigma_{j}^{h} f_{Q_{\sigma_{j}^{h}}(t, y)}\left|\nabla u_{j}(x)\right| \mathrm{d} x\right) \mathrm{d} t\right) \mathrm{d} \mathcal{H}^{n-1}(y)
$$

where $A_{y}^{\prime}=\left\{t \in \mathbb{R}:(t, y) \in A^{\prime}\right\}$ and $\tilde{A}^{\prime}=\left\{y \in \mathbb{R}^{n-1}: A_{y}^{\prime} \neq \emptyset\right\}$. In view of the definition (4.1) of $\tilde{Q}$, for every $(t, y) \in A^{\prime}$ :

$$
\begin{aligned}
f_{Q_{\sigma_{j}^{h}}(t, y)}\left|\nabla u_{j}(x)\right| \mathrm{d} x & \geq f_{Q_{\sigma_{j}^{h}(t, y)}}\left|\frac{\partial u_{j}}{\partial x_{1}}(x)\right| \mathrm{d} x \\
& \geq f_{t-\sigma_{j}^{h}}^{t+\sigma_{j}^{h}}\left|f_{\tilde{Q}_{\sigma_{j}^{h}(y)}} \frac{\partial u_{j}}{\partial s}(s, z) \mathrm{d} z\right| \mathrm{d} s ;
\end{aligned}
$$

Consider $u_{j}$ extended with value 0 outside $A$. Then, as in Lemma 4.4, for a.e. $s \in \mathbb{R}$ and for every $y \in \mathbb{R}^{n-1}$ we can define

$$
v_{j}^{y}(s)=v_{j}(s, y)=f_{\tilde{Q}_{\sigma_{j}^{h}}(y)} u_{j}(s, z) \mathrm{d} z .
$$

It turns out that if $y \in \tilde{A}^{\prime}$, then $v_{j}^{y} \in W^{1,1}\left(V_{y}\right)$ for a suitable open neighborhood $V_{y}$ of $\overline{A_{y}^{\prime}}$ (recall that $\left.A^{\prime} \subset \subset A\right)$; furthermore, (4.2) gives:

$$
f_{Q_{\sigma_{j}^{h}}(t, y)}\left|\nabla u_{j}(x)\right| \mathrm{d} x \geq f_{t-\sigma_{j}^{h}}^{t+\sigma_{j}^{h}}\left|\frac{d v_{j}^{y}}{\mathrm{~d} s}(s)\right| \mathrm{d} s .
$$

By Lemma 4.4 and Remark 4.5, the sequence $\left(v_{j}^{y}\right)$ converges (up to a subsequence, which does not affect the rest of the proof) to $u^{y}:=u(\cdot, y)$ in $L^{1}\left(V_{y}\right)$ for a.e. $y \in \tilde{A}^{\prime}$. By Fatou's Lemma:

$$
\liminf _{j \rightarrow+\infty} F_{\varepsilon_{j}}\left(u_{j}, A\right) \geq \int_{\tilde{A}^{\prime}} \frac{1}{h}\left(\liminf _{j \rightarrow+\infty} \frac{1}{\sigma_{j}^{h}} \int_{A_{y}^{\prime}} f^{h}\left(\sigma_{j}^{h} f_{t-\sigma_{j}^{h}}^{t+\sigma_{j}^{h}}\left|\frac{\mathrm{d} v_{j}^{y}}{\mathrm{~d} s}(s)\right| \mathrm{d} s\right) \mathrm{d} t\right) \mathrm{d} y
$$

thus, taking the one-dimensional $\Gamma$-convergence result (Rem. 3.5) into account:

$$
\liminf _{j \rightarrow+\infty} F_{\varepsilon_{j}}\left(u_{j}, A\right) \geq \int_{\tilde{A}^{\prime}} \frac{1}{h}\left(\int_{A_{y}^{\prime}} c_{h} h\left|\left(u^{y}\right)^{\prime}(s)\right| \mathrm{d} s+c_{h} h\left|D^{c} u^{y}\right|\left(A_{y}^{\prime}\right)\right) \mathrm{d} y .
$$

By Theorem 2.3 we deduce that

$$
\liminf _{j \rightarrow+\infty} F_{\varepsilon_{j}}\left(u_{j}, A\right) \geq c_{h}\left(\int_{A^{\prime}}\left|\left\langle\nabla u(x), e_{1}\right\rangle\right| \mathrm{d} x+\left|\left\langle D^{c} u, e_{1}\right\rangle\right|\left(A^{\prime}\right)\right) .
$$

As mentioned above, this result holds with any $\xi$ in place of $e_{1}$; therefore, since $c_{h} \rightarrow 1$ as $h \rightarrow+\infty$ and $A^{\prime} \subset \subset A$ is arbitrary, we get

$$
F^{\prime}(u, A) \geq \int_{A}|\langle\nabla u(x), \xi\rangle| \mathrm{d} x, \quad F^{\prime}(u, A) \geq\left|\left\langle D^{c} u, \xi\right\rangle\right|(A)
$$

for every $\xi \in S^{n-1}$. From the first inequality, the superadditivity of $F^{\prime}$ and Lemma 2.7 we get $F^{\prime}(u, A) \geq$ $\int_{A}|\nabla u(x)| \mathrm{d} x$ for every $A$. 
Let $\lambda=D^{c} u$ and $\lambda_{\xi}=\langle\lambda, \xi\rangle$; note that:

$$
\left|\lambda_{\xi}\right|=\left|\frac{\mathrm{d} \lambda_{\xi}}{\mathrm{d}|\lambda|}\right| \mathrm{d}|\lambda|=\left|\left\langle\frac{\mathrm{d} \lambda}{\mathrm{d}|\lambda|}, \xi\right\rangle\right| \mathrm{d}|\lambda| .
$$

The second inequality in (4.3) can now be rewritten as $F^{\prime}(u, A) \geq \int_{A} \psi_{\xi} \mathrm{d}|\lambda|$, where $\psi_{\xi}=|\langle\mathrm{d} \lambda / \mathrm{d}|\lambda|, \xi\rangle|$; an application of Lemma 2.7 yields:

$$
F^{\prime}(u, A) \geq \int_{A} \sup _{\xi}\left|\left\langle\frac{\mathrm{d} \lambda}{\mathrm{d}|\lambda|}, \xi\right\rangle\right| \mathrm{d}|\lambda| \geq \int_{A}\left|\sup _{\xi}\left\langle\frac{\mathrm{d} \lambda}{\mathrm{d}|\lambda|}, \xi\right\rangle\right| \mathrm{d}|\lambda|=|\lambda|(A)=\left|D^{c} u\right|(A) .
$$

This concludes the proof.

\section{LOWER BOUND IN TERMS OF THE SURFACE ENERGY}

In this section for any sequence $\left(F_{\varepsilon_{j}}\right)$ and any given function $u \in B V$ we apply Besicovitch's differentiation Theorem, with respect to $\mathcal{H}^{n-1}\left\llcorner S_{u}\right.$, to the (inner regular envelope of the) lower $\Gamma$-limit considered as a set function (Prop. 5.1). Through a rescaling argument the density of this bound will be estimated in terms of $F^{\prime}$ on the functions $u_{0}$ obtained by "blowing-up" $u$ around the jump points (Prop. 5.2 and subsequent corollary). We next need to express this bound in terms of $F^{\prime}\left(u_{0}, C_{1}^{\nu}\left(x_{0}\right)\right)$ where $C_{1}^{\nu}\left(x_{0}\right)$ is a cilinder of unit size with axis normal to the jump set in $x_{0}$ (Prop. 5.5): this allows an explicit computation of the lower bound (see Prop. 5.6 together with 5.7).

Denote by $\mathcal{A}(\Omega)$ the family of the open subsets of $\Omega$. Let $\left(\varepsilon_{j}\right)$ be a positive infinitesimal sequence and, for every $A \in \mathcal{A}(\Omega)$, let $\Phi^{\prime}(\cdot, A)$ be the lower $\Gamma$-limit of $\left(F_{\varepsilon_{j}}(\cdot, A)\right)$. We define the inner regular envelope of $\Phi^{\prime}$ as:

$$
\Phi_{-}^{\prime}(\cdot, A)=\sup \left\{\Phi^{\prime}(\cdot, B): B \in \mathcal{A}(\Omega), B \subset \subset A\right\} .
$$

It is easy to see that $\Phi_{-}^{\prime}(\cdot, A)$ is lower semicontinuous in $L^{1}(\Omega)$ for every $A$, and that $\Phi_{-}^{\prime}(u, \cdot)$ is superadditive for every $u$.

Proposition 5.1. For every $u \in L^{1}(\Omega)$ the function $\Phi_{-}^{\prime}(u, \cdot): \mathcal{A}(\Omega) \rightarrow \mathbb{R}$ is the trace on $\mathcal{A}(\Omega)$ of a Borel measure on $\Omega$; moreover, for every $A \in \mathcal{A}(\Omega)$ and $u \in B V(\Omega)$ :

$$
\Phi_{-}^{\prime}(u, A) \geq \int_{S_{u} \cap A} h(x) \mathrm{d} \mathcal{H}^{n-1},
$$

where, for $\mathcal{H}^{n-1}$ a.e. $x \in S_{u}$ :

$$
h(x)=\lim _{\varrho \rightarrow 0} \frac{\Phi_{-}^{\prime}\left(u, B_{\varrho}(x)\right)}{\omega_{n-1} \varrho^{n-1}} .
$$

Proof. An increasing set function $\alpha: \mathcal{A}(\Omega) \rightarrow[0,+\infty]$, which satisfies $\alpha(0)=0$ and which is subadditive, superadditive and inner regular, can be extended to a Borel measure on $\Omega$ : see, e.g. [19], Theorem 14.23. The result can be applied to $\Phi_{-}^{\prime}$ if we check the subadditivity: this can be shown following the proof of Proposition 4.3 and Theorem 4.6 of [14] (these results are established in the case $p>1$, but the same arguments work if $p=1$ ).

Given $u \in B V(\Omega)$, for every $k \in \mathbb{N}$ let $S_{k}=\left\{x \in S_{u}:\left|u^{+}(x)-u^{-}(x)\right|>1 / k\right\}$. Clearly, $\mathcal{H}^{n-1}\left(S_{k}\right)<+\infty$; let $\nu_{k}=\mathcal{H}^{n-1}\left\llcorner S_{k}\right.$, and denote by $\mu$ the Borel measure which extends $\Phi_{-}^{\prime}(u, \cdot)$ : in view of Remark 3.7, $\mu$ is a finite measure. By Besicovitch's differentiation Theorem (see, e.g. [27], Th. 4.7), the limit

$$
g(x)=\lim _{\varrho \rightarrow 0} \frac{\mu\left(B_{\varrho}(x)\right)}{\nu_{k}\left(B_{\varrho}(x)\right)}
$$


exists and is finite for $\nu_{k}$-a.e. $x \in \Omega$, and is $\nu_{k}$-measurable; moreover, the Radon-Nikodym decomposition of $\mu$ is given by $\mu=g \nu_{k}+\mu^{s}$, with $\mu^{s} \perp \nu_{k}$. Since $S_{k}$ is $\mathcal{H}^{n-1}$-rectifiable, for $\mathcal{H}^{n-1}$-a.e. $x \in S_{k}$ we have

$$
\frac{\nu_{k}\left(B_{\varrho}(x)\right)}{\omega_{n-1} \varrho^{n-1}}=\frac{\mathcal{H}^{n-1}\left(B_{\varrho}(x) \cap S_{k}\right)}{\omega_{n-1} \varrho^{n-1}} \rightarrow 1, \quad \varrho \rightarrow 0
$$

(see, e.g., [5] Th. 2.63). Thus, for $\mathcal{H}^{n-1}$-a.e. $x \in S_{k}$

$$
g(x)=\lim _{\varrho \rightarrow 0} \frac{\mu\left(B_{\varrho}(x)\right)}{\omega_{n-1} \varrho^{n-1}}=\lim _{\varrho \rightarrow 0} \frac{\Phi_{-}^{\prime}\left(u, B_{\varrho}(x)\right)}{\omega_{n-1} \varrho^{n-1}}=h(x) .
$$

Taking into account that $\mu^{s}$ is non-negative, we deduce that for every $A \in \mathcal{A}(\Omega)$

$$
\Phi_{-}^{\prime}(u, A) \geq \int_{A} h(x) \mathrm{d} \nu_{k}=\int_{S_{k} \cap A} h(x) \mathrm{d} \mathcal{H}^{n-1} .
$$

The conclusion follws by considering the supremum for $k \in \mathbb{N}$.

When considering $F^{\prime}$ for the blow-up $u_{0}$ on a unit ball $B_{1}$ as below (or on a cilinder $C_{1}$ of unit size, as in Prop. 5.5), we assume as $\Omega$ any set strictly containing $B_{1}$ (or $C_{1}$ ): see Remark 3.4.

Proposition 5.2. For every $u \in B V(\Omega)$

$$
\liminf _{\varrho \rightarrow 0} \frac{\Phi_{-}^{\prime}\left(u, B_{\varrho}\left(x_{0}\right)\right)}{\varrho^{n-1}} \geq F^{\prime}\left(u_{0}, B_{1}\left(x_{0}\right)\right), \quad \text { for } \mathcal{H}^{n-1} \text {-a.e. } \quad x_{0} \in S_{u},
$$

where $u_{0}$ is the function given by

$$
u_{0}(x)= \begin{cases}u^{+}\left(x_{0}\right) & \text { if }\left\langle x-x_{0}, \nu\right\rangle \geq 0 \\ u^{-}\left(x_{0}\right) & \text { if }\left\langle x-x_{0}, \nu\right\rangle<0\end{cases}
$$

with $\nu=\nu_{u}\left(x_{0}\right)$

Proof. Let $\delta \in(0,1)$ and note that $\Phi_{-}^{\prime}\left(u, B_{\varrho}\left(x_{0}\right)\right) \geq \Phi^{\prime}\left(u, B_{\delta \varrho}\left(x_{0}\right)\right)$ for every $\varrho$; then

$$
\liminf _{\varrho \rightarrow 0} \frac{\Phi_{-}^{\prime}\left(u, B_{\varrho}\left(x_{0}\right)\right)}{\varrho^{n-1}} \geq \delta^{n-1} \liminf _{r \rightarrow 0} \frac{\Phi^{\prime}\left(u, B_{r}\left(x_{0}\right)\right)}{r^{n-1}} .
$$

Let us now estimate the lower limit in the right-hand side.

We can assume $x_{0}=0$. Let $\left(r_{k}\right)$ be a decreasing infinitesimal sequence; for every $k \in \mathbb{N}$ there exists $w_{j} \in W^{1,1}(\Omega)$ such that $w_{j} \rightarrow u$ in $L^{1}(\Omega)$ and

$$
\liminf _{j \rightarrow+\infty} F_{\varepsilon_{j}}\left(w_{j}, B_{r_{k}}(0)\right) \leq \Phi^{\prime}\left(u, B_{r_{k}}(0)\right)+\frac{r_{k}^{n-1}}{2 k} .
$$

Let $\bar{j}=j(k)$ be such that $\varepsilon_{\bar{j}} / r_{k} \leq 1 / k$ and

$$
\begin{gathered}
F_{\varepsilon_{\bar{j}}}\left(w_{\bar{j}}, B_{r_{k}}(0)\right) \leq \Phi^{\prime}\left(u, B_{r_{k}}(0)\right)+\frac{r_{k}^{n-1}}{k} \\
\left\|w_{\bar{j}}-u\right\|_{L^{1}(\Omega)} \leq \frac{1}{k}, \quad \int_{B_{2}(0)}\left|w_{\bar{j}}\left(r_{k} x\right)-u\left(r_{k} x\right)\right| \mathrm{d} x \leq \frac{1}{k} .
\end{gathered}
$$


Let $u_{k}=w_{j(k)}$. We can suppose that the sequence $(j(k))$ is increasing, and we set $\sigma_{k}=\varepsilon_{j(k)}$; then $u_{k} \rightarrow u$ in $L^{1}(\Omega)$ and

$$
F_{\sigma_{k}}\left(u_{k}, B_{r_{k}}(0)\right) \leq \Phi^{\prime}\left(u, B_{r_{k}}(0)\right)+\frac{r_{k}^{n-1}}{k}, \quad \int_{B_{2}(0)}\left|u_{k}\left(r_{k} x\right)-u\left(r_{k} x\right)\right| \mathrm{d} x \leq \frac{1}{k} .
$$

The first inequality gives:

$$
\liminf _{k \rightarrow+\infty} \frac{\Phi^{\prime}\left(u, B_{r_{k}}(0)\right)}{r_{k}^{n-1}} \geq \liminf _{k \rightarrow+\infty} \frac{F_{\sigma_{k}}\left(u_{k}, B_{r_{k}}(0)\right)}{r_{k}^{n-1}} ;
$$

while, from the second inequality and the definition (2.1) of $u^{ \pm}$we obtain:

$$
\int_{B_{2}(0)}\left|u_{k}\left(r_{k} x\right)-u_{0}\left(r_{k} x\right)\right| \mathrm{d} x \leq \frac{1}{k}+\int_{B_{2}(0)}\left|u\left(r_{k} x\right)-u_{0}\left(r_{k} x\right)\right| \mathrm{d} x \stackrel{k}{\rightarrow} 0 .
$$

If we define $v_{k}(t)=u_{k}\left(r_{k} t\right)$, then $v_{k} \rightarrow u_{0}$ in $L^{1}\left(B_{2}(0)\right)$; moreover, for every $x \in B_{r_{k}}(0)$

$$
f_{B_{\sigma_{k}}(x)}\left|\nabla u_{k}(y)\right| \mathrm{d} y=\frac{r_{k}^{n-1}}{\omega_{n} \sigma_{k}^{n}} \int_{B_{\sigma_{k} / r_{k}}\left(x / r_{k}\right)}\left|\nabla v_{k}(t)\right| \mathrm{d} t .
$$

Therefore, setting $x / r_{k}=z$, we have:

$$
\frac{F_{\sigma_{k}}\left(u_{k}, B_{r_{k}}(0)\right)}{r_{k}^{n-1}}=\frac{1}{\sigma_{k} / r_{k}} \int_{B_{1}(0)} f\left(\frac{\sigma_{k}}{r_{k}} f_{B_{\sigma_{k} / r_{k}}(z)}\left|\nabla v_{k}(t)\right| \mathrm{d} t\right) \mathrm{d} z
$$

Since $\gamma_{k}:=\sigma_{k} / r_{k} \rightarrow 0$, and $v_{k} \rightarrow u_{0}$ in $L^{1}\left(B_{2}(0)\right)$, by the arbitrariness of $\left(r_{k}\right)$ and the definition of $F^{\prime}$, we conclude that

$$
\liminf _{r \rightarrow 0} \frac{\Phi^{\prime}\left(u, B_{r}(0)\right)}{r^{n-1}} \geq F^{\prime}\left(u_{0}, B_{1}(0)\right) .
$$

This, together with (5.1) and the arbitrariness of $\delta$, yields the conclusion.

From the two propositions we have just proved the next result immediately follows.

Corollary 5.3. Let $u \in B V(\Omega)$ and let $A$ be an open subset of $\Omega$. Then

$$
F^{\prime}(u, A) \geq \int_{S_{u} \cap A} \sigma(x) \mathrm{d} \mathcal{H}^{n-1},
$$

where

with $\left(\nu=\nu_{u}\left(x_{0}\right)\right)$

$$
\sigma\left(x_{0}\right)=\omega_{n-1}^{-1} F^{\prime}\left(u_{0}, B_{1}\left(x_{0}\right)\right), \quad \text { for } \mathcal{H}^{n-1} \text {-a.e. } x_{0} \in S_{u}
$$

$$
u_{0}(x)= \begin{cases}u^{+}\left(x_{0}\right) & \text { if }\left\langle x-x_{0}, \nu\right\rangle \geq 0 \\ u^{-}\left(x_{0}\right) & \text { if }\left\langle x-x_{0}, \nu\right\rangle<0\end{cases}
$$

Let $\nu \in S^{n-1}$. For any $y \in \mathbb{R}^{n}$ denote by $y_{\nu}$ and $y_{\nu^{\perp}}$ the projections onto the subspaces $V=\{t \nu: t \in \mathbb{R}\}$ and $V^{\perp}$, respectively. For $\varrho>0$ and $x \in \mathbb{R}^{n}$ define

$$
C_{\varrho}^{\nu}(0)=\left\{y \in \mathbb{R}^{n}:\left|y_{\nu}\right|<\varrho,\left|y_{\nu}\right|<\varrho\right\}, \quad C_{\varrho}^{\nu}(x)=x+C_{\varrho}^{\nu}(0) .
$$

The next lemma proves that the "transition set" between two constant values shrinks onto the interface. 
Lemma 5.4. Let $\nu \in S^{n-1}$ and

$$
u_{0}(x)= \begin{cases}a & \text { if }\langle x, \nu\rangle \geq 0 \\ b & \text { if }\langle x, \nu\rangle<0\end{cases}
$$

Let $\Omega^{\prime} \supset \supset C_{1}^{\nu}(0)$. For any $A$ open subset of $C_{1}^{\nu}(0)$, there exist a positive infinitesimal sequence $\left(\varepsilon_{j}\right)$ and a sequence $\left(u_{j}\right)$ in $W^{1,1}\left(\Omega^{\prime}\right)$ converging to $u_{0}$ in $L^{1}\left(\Omega^{\prime}\right)$ and such that

$$
\begin{gathered}
\lim _{j \rightarrow+\infty} F_{\varepsilon_{j}}\left(u_{j}, A\right)=F^{\prime}\left(u_{0}, A\right), \\
u_{j}(x)=a \quad \text { if }\langle x, \nu\rangle \geq a_{j} ; \quad u_{j}(x)=b \quad \text { if }\langle x, \nu\rangle \leq-b_{j},
\end{gathered}
$$

where $\left(a_{j}\right)$ and $\left(b_{j}\right)$ are suitable positive infinitesimal sequences.

Proof. It is not restrictive to assume $\nu=e_{1}$. Fix $A$ open subset of $C_{1}^{\nu}(0)$.

Step 1. Let $0<\varepsilon<d\left(C_{1}^{\nu}(0), \partial \Omega^{\prime}\right)$, and $\sigma>0$. Let $\varphi$ be the continuous function given by

$$
\varphi(x)= \begin{cases}0 & \text { if } x_{1} \leq-2 \varepsilon-\sigma \\ \text { affine } & \text { if }-2 \varepsilon-\sigma<x_{1}<-2 \varepsilon \\ 1 & \text { if } x_{1} \geq-2 \varepsilon\end{cases}
$$

Clearly, $|\nabla \varphi| \leq 1 / \sigma$. Define:

$$
\begin{gathered}
A_{\varepsilon}=\left\{x \in \mathbb{R}^{n}: x_{1}<-3 \varepsilon-\sigma\right\}, \quad B_{\varepsilon}=\left\{x \in \mathbb{R}^{n}: x_{1}>-\varepsilon\right\}, \\
S_{\varepsilon}=\left\{x \in \mathbb{R}^{n}:-3 \varepsilon-\sigma<x_{1}<-\varepsilon\right\} .
\end{gathered}
$$

Let $u_{1}, u_{2} \in W^{1,1}\left(\Omega^{\prime}\right)$ and $v=\varphi u_{1}+(1-\varphi) u_{2}$; then

$$
\begin{aligned}
\varepsilon F_{\varepsilon}(v, A)=\int_{A \cap A_{\varepsilon}} f\left(\varepsilon f_{B_{\varepsilon}(x)}\left|\nabla u_{2}(y)\right| \mathrm{d} y\right) \mathrm{d} x & \\
& +\int_{A \cap B_{\varepsilon}} f\left(\varepsilon f_{B_{\varepsilon}(x)}\left|\nabla u_{1}(y)\right| \mathrm{d} y\right) \mathrm{d} x+\int_{A \cap S_{\varepsilon}} f\left(\varepsilon f_{B_{\varepsilon}(x)}|\nabla v(y)| \mathrm{d} y\right) \mathrm{d} x .
\end{aligned}
$$

By the subadditivity of $f$,

$$
\begin{aligned}
\int_{A \cap S_{\varepsilon}} f(\varepsilon & \left.f_{B_{\varepsilon}(x)}|\nabla v(y)| \mathrm{d} y\right) \leq \int_{A \cap S_{\varepsilon}} f\left(\varepsilon f _ { B _ { \varepsilon } ( x ) } \left[\varphi(y)\left|\nabla u_{1}(y)\right|+(1-\varphi(y))\left|\nabla u_{2}(y)\right|\right.\right. \\
& \left.\left.+\left|u_{1}(y)-u_{2}(y)\right||\nabla \varphi(y)|\right] \mathrm{d} y\right) \mathrm{d} x \leq \int_{A \cap S_{\varepsilon}} f\left(\varepsilon f_{B_{\varepsilon}(x)} \varphi(y)\left|\nabla u_{1}(y)\right| \mathrm{d} y\right) \mathrm{d} x \\
& +\int_{A \cap S_{\varepsilon}} f\left(\varepsilon f_{B_{\varepsilon}(x)}(1-\varphi(y))\left|\nabla u_{2}(y)\right| \mathrm{d} y\right) \mathrm{d} x+\int_{A \cap S_{\varepsilon}} f\left(\varepsilon f_{B_{\varepsilon}(x)}\left|u_{1}(y)-u_{2}(y)\right||\nabla \varphi(y)| \mathrm{d} y\right) \mathrm{d} x
\end{aligned}
$$

Then, since $f(t) \leq t$,

$$
F_{\varepsilon}(v, A) \leq F_{\varepsilon}\left(u_{1}, A \cap\left(B_{\varepsilon} \cup S_{\varepsilon}\right)\right)+F_{\varepsilon}\left(u_{2}, A \cap\left(A_{\varepsilon} \cup S_{\varepsilon}\right)\right)+I_{\varepsilon}
$$

with

$$
I_{\varepsilon} \leq \frac{1}{\sigma} \int_{C_{1}^{\nu}(0) \cap S_{\varepsilon}} f_{B_{\varepsilon}(x)}\left|u_{1}(y)-u_{2}(y)\right| \mathrm{d} y \mathrm{~d} x
$$


A simple application of Fubini's theorem now gives

$$
I_{\varepsilon} \leq \frac{1}{\sigma} \int_{\Omega_{-}^{\prime}}\left|u_{1}(y)-u_{2}(y)\right| \mathrm{d} y,
$$

where $\Omega_{-}^{\prime}=\left\{x \in \Omega^{\prime}: x_{1}<0\right\}$.

Step 2. Let $\left(\varepsilon_{j}\right)$ be a positive infinitesimal sequence, and $\left(u_{j}\right)$ in $W^{1,1}\left(\Omega^{\prime}\right)$ converging to $u_{0}$ in $L^{1}\left(\Omega^{\prime}\right)$ such that $F_{\varepsilon_{j}}\left(u_{j}, A\right) \rightarrow F^{\prime}\left(u_{0}, A\right)$. For every $j \in \mathbb{N}$ apply estimate (5.3) with $\varepsilon=\varepsilon_{j}, u_{1}=u_{j}$ and $u_{2}=b$. If $v_{j}=\varphi u_{j}+(1-\varphi) b$, since $F_{\varepsilon_{j}}(b, A)=0$, we have:

$$
F_{\varepsilon_{j}}\left(v_{j}, A\right) \leq F_{\varepsilon_{j}}\left(u_{j}, A\right)+\frac{1}{\sigma} \int_{\Omega_{-}^{\prime}}\left|u_{j}(y)-b\right| \mathrm{d} y .
$$

Note that

$$
\int_{\Omega_{-}^{\prime}}\left|u_{j}(y)-b\right| \mathrm{d} y=\int_{\Omega_{-}^{\prime}}\left|u_{j}(y)-u_{0}(y)\right| \mathrm{d} y \stackrel{j}{\rightarrow} 0 .
$$

Therefore, if $\left(\sigma_{h}\right)$ is a positive infinitesimal sequence, we can find a strictly increasing sequence $\left(j_{h}\right)$ in $\mathbb{N}$ such that

Then

$$
\frac{1}{\sigma_{h}} \int_{\Omega_{-}^{\prime}}\left|u_{j_{h}}-b\right| \mathrm{d} y \stackrel{h}{\rightarrow} 0 .
$$

Moreover,

$$
\limsup _{h \rightarrow+\infty} F_{\varepsilon_{j_{h}}}\left(v_{j_{h}}, A\right) \leq \limsup _{h \rightarrow+\infty} F_{\varepsilon_{j_{h}}}\left(u_{j_{h}}, A\right)=F^{\prime}\left(u_{0}, A\right) .
$$

$$
v_{j_{h}}(x)= \begin{cases}b & x_{1} \leq-2 \varepsilon_{j_{h}}-\sigma_{h} \\ u_{j_{h}} & x_{1} \geq 0\end{cases}
$$

Clearly $v_{j_{h}} \rightarrow u_{0}$ in $L^{1}\left(\Omega^{\prime}\right)$, and $\lim \inf F_{\varepsilon_{j_{h}}}\left(v_{j_{h}}, A\right) \geq F^{\prime}\left(u_{0}, A\right)$. An analogous procedure allows to modify $v_{j_{h}}$ so that, while mantaining the convergence of the functionals to $F^{\prime}\left(u_{0}, A\right)$, it takes the value $a$ at the points $x$ with $x_{1}$ positive and outside a neighborhood of 0 , shrinking to 0 .

Proposition 5.5. For $\mathcal{H}^{n-1}$ a.e. $x_{0} \in S_{u}$

$$
F^{\prime}\left(u_{0}, B_{1}\left(x_{0}\right)\right)=F^{\prime}\left(u_{0}, C_{1}^{\nu}\left(x_{0}\right)\right)
$$

where $\nu=\nu_{u}\left(x_{0}\right)$ and $u_{0}$ is as (5.2).

Proof. Clearly, the non-trivial inequality is $F^{\prime}\left(u_{0}, B_{1}\left(x_{0}\right)\right) \geq F^{\prime}\left(u_{0}, C_{1}^{\nu}\left(x_{0}\right)\right)$. It is not restrictive to assume $x_{0}=0$ and $\nu=e_{1}$. Fix $0<\delta<1$. Let $\left(\varepsilon_{j}\right)$ and $\left(u_{j}\right)$ be given by the previous lemma, applied with $A=B_{1}(0)$. Then $u_{j}(x)=a$ if $x_{1} \geq a_{j}$, and $u_{j}(x)=b$ if $x_{1} \leq-b_{j}$, where $\left(a_{j}\right)$ and $\left(b_{j}\right)$ are suitable positive infinitesimal sequences. Let $S_{j}=\left(-b_{j}, a_{j}\right) \times \mathbb{R}^{n-1}$; then, for $j$ sufficiently large, we have $C_{\delta}^{\nu}(0) \cap S_{j} \subset \subset B_{1}(0)$, and therefore $F_{\varepsilon_{j}}\left(u_{j}, C_{\delta}^{\nu}(0) \cap B_{1}(0)\right)=F_{\varepsilon_{j}}\left(u_{j}, C_{\delta}^{\nu}(0)\right)$. Hence:

$$
F_{\varepsilon_{j}}\left(u_{j}, B_{1}(0)\right) \geq F_{\varepsilon_{j}}\left(u_{j}, B_{1}(0) \cap C_{\delta}^{\nu}(0)\right)=F_{\varepsilon_{j}}\left(u_{j}, C_{\delta}^{\nu}(0)\right) .
$$

Moreover, if we set $v_{j}(x)=u_{j}(\delta x)$, then $F_{\varepsilon_{j}}\left(u_{j}, C_{\delta}^{\nu}(0)\right)=\delta^{n-1} F_{\varepsilon_{j} / \delta}\left(v_{j}, C_{1}^{\nu}(0)\right)$, as one can easily check by a change of variable. Thus, passing to the limit in (5.4), we get $F^{\prime}\left(u_{0}, B_{1}(0)\right) \geq \delta^{n-1} \liminf _{j} F_{\varepsilon_{j} / \delta}\left(v_{j}, C_{1}^{\nu}(0)\right) \geq$ $\delta^{n-1} F^{\prime}\left(u_{0}, C_{1}^{\nu}(0)\right)$. The conclusion now follows by taking the supremum on $\delta \in(0,1)$. 
Proposition 5.6. Let $u_{0}$ be as in (5.2), with $a \neq b$ and $\nu=e_{1}$. Then

$$
F^{\prime}\left(u_{0}, C_{1}^{e_{1}}(0)\right) \geq \omega_{n-1} \inf _{X} G
$$

where

$$
G(v)=\int_{\mathbb{R}} f\left(a_{n} \int_{0}^{1}\left[v\left(\xi+\sqrt{1-\eta^{2}}, \eta\right)-v\left(\xi-\sqrt{1-\eta^{2}}, \eta\right)\right] \eta^{n-2} \mathrm{~d} \eta\right) \mathrm{d} \xi,
$$

$a_{n}=(n-1) \omega_{n-1} / \omega_{n}$, and $X$ is the space of all functions $v \in W_{l o c}^{1,1}(\mathbb{R} \times(0,1))$ which are non-decreasing in the first variable, such that there exists $\xi_{0}<\xi_{1}$ (depending on $v$ ) with $v(\xi, \eta)=b$ if $\xi \leq \xi_{0}$, and $v(\xi, \eta)=a$ if $\xi \geq \xi_{1}$.

Proof. Denote $C_{1}^{e_{1}}(0)$ by $C$. We can suppose $a>0$ and $b=0$.

Let $\left(\varepsilon_{j}\right)$ and $\left(u_{j}\right)$ be the sequences given by Lemma 5.4, applied with $A=C$ and any $\Omega^{\prime} \supset \supset$. In particular, $u_{j}(x)=0$ if $x_{1} \leq-b_{j}$, and $u_{j}(x)=a$ if $x_{1} \geq a_{j}$, for some positive $a_{j}, b_{j} \rightarrow 0$.

Let

$$
\alpha_{j}\left(x_{1}, x_{2}, \ldots, x_{n}\right)=\int_{-1}^{x_{1}}\left(\frac{\partial}{\partial t} u_{j}\left(t, x_{2}, \ldots, x_{n}\right)\right)^{+} \mathrm{d} t
$$

and $v_{j}=\alpha_{j} \wedge a$. Then $v_{j} \in W^{1,1}\left(\Omega^{\prime}\right), v_{j}(x)=u_{0}(x)$ if $x_{1} \notin\left(-b_{j}, a_{j}\right)$, and $v_{j}$ is non-decreasing in the first variable. Moreover

Then, for $j$ sufficiently large,

$$
\left|\nabla u_{j}\right| \geq\left|\frac{\partial u_{j}}{\partial x_{1}}\right| \geq \frac{\partial v_{j}}{\partial x_{1}} \geq 0
$$

$$
F_{\varepsilon_{j}}\left(u_{j}, C\right) \geq \frac{1}{\varepsilon_{j}} \int_{C} f\left(\varepsilon_{j} f_{B_{\varepsilon_{j}}(x)} \frac{\partial v_{j}}{\partial s_{1}}(s) \mathrm{d} s\right) \mathrm{d} x .
$$

Let $B_{\varrho}^{n-1}$ be the $(n-1)$-dimensional ball of centre 0 and radius $\varrho$. Since $C=(-1,1) \times B_{1}^{n-1}$, we have

$$
F_{\varepsilon_{j}}\left(u_{j}, C\right) \geq \frac{1}{\varepsilon_{j}} \int_{B_{1}^{n-1}} \mathrm{~d} x_{2} \cdots \mathrm{d} x_{n} \int_{-1}^{1} f\left(\varepsilon_{j} f_{B_{\varepsilon_{j}}(x)} \frac{\partial v_{j}}{\partial s_{1}}(s) \mathrm{d} s\right) \mathrm{d} x_{1} .
$$

Let $\left(\bar{x}_{2}, \cdots, \bar{x}_{n}\right)$ be in the closure $\bar{B}_{1}^{n-1}$ of $B_{1}^{n-1}$ be such that

$$
\int_{-1}^{1} f\left(\varepsilon_{j} f_{B_{\varepsilon_{j}}\left(x_{1}, \bar{x}_{2}, \cdots, \bar{x}_{n}\right)} \frac{\partial v_{j}}{\partial s_{1}}(s) \mathrm{d} s\right) \mathrm{d} x_{1}=\min _{\left(x_{2}, \cdots, x_{n}\right) \in \bar{B}_{1}^{n-1}} \int_{-1}^{1} f\left(\varepsilon_{j} f_{B_{\varepsilon_{j}}\left(x_{1}, \cdots, x_{n}\right)} \frac{\partial v_{j}}{\partial s_{1}}(s) \mathrm{d} s\right) \mathrm{d} x_{1} .
$$

In the argument below it will not be restrictive to assume $\bar{x}_{i}=0$ for every $i$. Then

$$
F_{\varepsilon_{j}}\left(u_{j}, C\right) \geq \frac{\omega_{n-1}}{\varepsilon_{j}} \int_{-1}^{1} f\left(\frac{1}{\omega_{n} \varepsilon_{j}^{n-1}} \int_{B_{\varepsilon_{j}}\left(x_{1}, 0\right)} \frac{\partial v_{j}}{\partial s_{1}}(s) \mathrm{d} s\right) \mathrm{d} x_{1} .
$$

By Fubini's Theorem,

$$
\int_{B_{\varepsilon_{j}}\left(x_{1}, 0\right)} \frac{\partial v_{j}}{\partial s_{1}}(s) \mathrm{d} s=\int_{B_{\varepsilon_{j}}^{n-1}}\left[v_{j}\left(x_{1}+\sqrt{\varepsilon_{j}^{2}-|y|^{2}}, y\right)-v_{j}\left(x_{1}-\sqrt{\varepsilon_{j}^{2}-|y|^{2}}, y\right)\right] \mathrm{d} y .
$$

Define

$$
\hat{v}_{j}(t, \varrho)=f_{\partial B_{\varrho}^{n-1}} v_{j}(t, y) \mathrm{d} \mathcal{H}^{n-2}(y), \quad \varrho \in\left(0, \varepsilon_{j}\right)
$$


Then

$$
\begin{aligned}
\int_{B_{\varepsilon_{j}}\left(x_{1}, 0\right)} \frac{\partial v_{j}}{\partial s_{1}}(s) \mathrm{d} s=\int_{0}^{\varepsilon_{j}} \mathrm{~d} \varrho \int_{\partial B_{\varrho}^{n-1}}\left[v_{j}\right. & \left.\left(x_{1}+\sqrt{\varepsilon_{j}^{2}-|y|^{2}}, y\right)-v_{j}\left(x_{1}-\sqrt{\varepsilon_{j}^{2}-|y|^{2}}, y\right)\right] \mathrm{d} \mathcal{H}^{n-2}(y) \\
= & \int_{0}^{\varepsilon_{j}} \mathcal{H}^{n-2}\left(\partial B_{\varrho}^{n-1}\right)\left[\hat{v}_{j}\left(x_{1}+\sqrt{\varepsilon_{j}^{2}-\varrho^{2}}, \varrho\right)-\hat{v}_{j}\left(x_{1}-\sqrt{\varepsilon_{j}^{2}-\varrho^{2}}, \varrho\right)\right] \mathrm{d} \varrho .
\end{aligned}
$$

By the change of variables $x_{1} / \varepsilon_{j}=\xi$ and $\varrho=\varepsilon_{j} \eta$, we obtain, for $j$ sufficiently large:

$$
\begin{gathered}
F_{\varepsilon_{j}}\left(u_{j}, C\right) \geq \omega_{n-1} \int_{-1 / \varepsilon_{j}}^{1 / \varepsilon_{j}} f\left(\frac { ( n - 1 ) \omega _ { n - 1 } } { \omega _ { n } } \int _ { 0 } ^ { 1 } \left[\hat{v}_{j}\left(\varepsilon_{j} \xi+\varepsilon_{j} \sqrt{1-\eta^{2}}, \varepsilon_{j} \eta\right)\right.\right. \\
\left.\left.-\hat{v}_{j}\left(\varepsilon_{j} \xi-\varepsilon_{j} \sqrt{1-\eta^{2}}, \varepsilon_{j} \eta\right)\right] \eta^{n-2} \mathrm{~d} \eta\right) \mathrm{d} \xi .
\end{gathered}
$$

Let $w_{j}(x)=\hat{v}_{j}\left(\varepsilon_{j} x\right)$. Clearly $w_{j}$ is non-decreasing in the first variable, and there exist $\xi_{0}<\xi_{1}$ such that $w_{j}(x)=a$ if $x \geq \xi_{1}$ and $w_{j}(x)=0$ if $x \leq \xi_{0}$. Then $w_{j}$ can be extended to all $\mathbb{R} \times(0,1)$ (with values 0 and $a$ ) and thus $w_{j} \in X$. Hence $F^{\prime}\left(u_{0}, C\right) \geq \omega_{n-1} G\left(w_{j}\right)$.

We now need a precise estimate of the infimum of the functional $G$ introduced in Proposition 5.6. To bypass the problem of the existence of the minimum of $G$ we consider a discrete version $G_{k}(k \in \mathbb{N})$ of $G$. This leads to a minimum problem in a finite dimensional space, and a careful analysis of the properties of the minimizers allows to compute explicitely the infimum.

Proposition 5.7. With the notation of Proposition 5.6 we have

$$
\inf _{X} G \geq \vartheta(|a-b|)
$$

where $\vartheta$ is defined in $(3.2)$.

Proof. We can suppose $a>0$ and $b=0$. For each $k \in \mathbb{N}$ we now consider a discrete version $G_{k}$ of $G$ defined on the space of the functions on $S=\mathbb{R} \times[0,1]$ which are constant on each of the squares determined by a coordinate partition of $S$ and with sides of length $1 / k$. We also require the monotonicity in the first variable and the constant value 0 and $a$ on the left and right of $\left[\xi_{0}, \xi_{1}\right] \times[0,1]$, respectively, for some $\xi_{0}<\xi_{1}$.

Clearly, we can deal only with the values on the nodes; thus, for any $N \in \mathbb{N}$, we define $Y_{k}^{N}$ as the set of the functions

$$
v=\left(v^{i, j}\right)_{i, j}: \mathbb{Z} \times\{1, \ldots, k-1\} \rightarrow[0, a],
$$

such that:

a) for every $j$ the function $i \mapsto v^{i, j}$ is increasing;

b) $v^{i, j}=0$ if $i<-N k$ and $v^{i, j}=a$ if $i \geq N k$.

We think $v^{i, j}$ as the value in $(i / k, j / k)$ of the input function of $G$. Let $Y_{k}=\bigcup_{N \in \mathbb{N}} Y_{k}^{N}$, and let $G_{k}: Y_{k} \rightarrow \mathbb{R}$ be defined by:

$$
G_{k}(v)=\sum_{i \in \mathbb{Z}} \frac{1}{k} f\left(a_{n} \sum_{j=1}^{k-1} \frac{1}{k}[v]_{i, j}\right),
$$

where

$$
[v]_{i, j}=\left(v^{i+\hat{j}, j}-v^{i-\hat{j}, j}\right)(j / k)^{n-2},
$$


with $\hat{j}$ denoting the integer part of $\sqrt{1-(j / k)^{2}} /(1 / k)=\sqrt{k^{2}-j^{2}}$ (see Fig. 1 below).

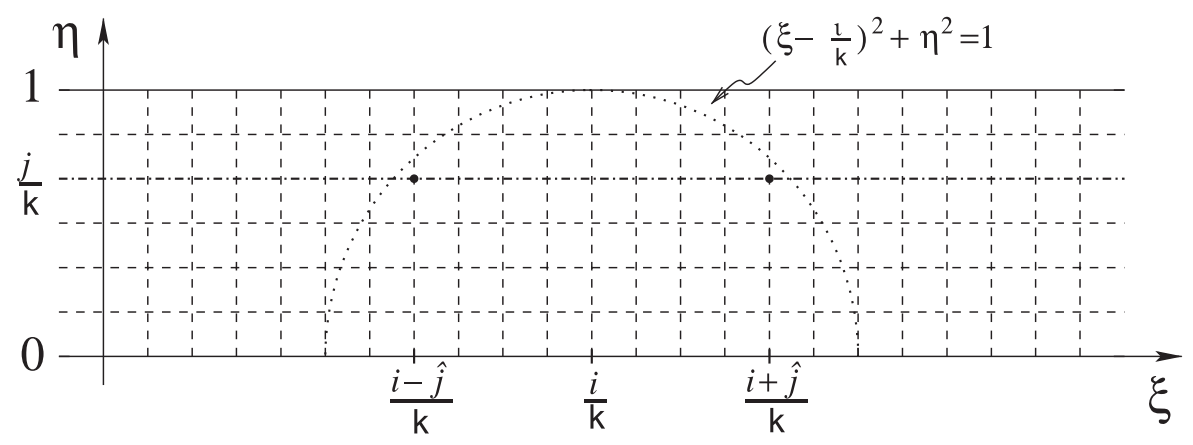

Figure 1. A sketch for the definition of $[v]_{i, j}$.

Note that:

$$
G_{k}(v)=\sum_{i=-(N+1) k}^{(N+1) k} \frac{1}{k} f\left(a_{n} \sum_{j=1}^{k-1} \frac{1}{k}[v]_{i, j}\right), \quad \text { on } Y_{k}^{N}
$$

Step 1. Each minimizer of $G_{k}$ on $Y_{k}^{N}$ takes only the values 0 and $a$.

Let $v$ be a minimizer of $G_{k}$ on $Y_{k}^{N}$. Suppose, by contradiction, that there exists $i_{0}, j_{0}$ with $v^{i_{0}, j_{0}}=c \in(0, a)$. We can assume that for a suitable $s \in \mathbb{N}$ :

$$
v^{i_{0}-1, j_{0}}<c, \quad c=v^{i_{0}, j_{0}}=v^{i_{0}+1, j_{0}}=\cdots=v^{i_{0}+s, j_{0}}, \quad v^{i_{0}+s+1, j_{0}}>c .
$$

Then, given $t \in \mathbb{R}$, we define $v_{t}=\left(v_{t}^{i, j}\right)_{i, j}$ as $v_{t}^{i_{0}+l, j_{0}}=c+t$, if $0 \leq l \leq s$, and $v_{t}=v$ otherwise. For $|t|$ sufficiently small, $v_{t} \in Y_{k}^{N}$. Let

$$
I_{1}=\left\{i \in \mathbb{Z}: i+\hat{j}_{0} \in\left[i_{0}, i_{0}+s\right]\right\}, \quad I_{2}=\left\{i \in \mathbb{Z}: i-\hat{j}_{0} \in\left[i_{0}, i_{0}+s\right]\right\} .
$$

Note that if $i \notin I_{1} \Delta I_{2}$ then $\left[v_{t}\right]_{i, j}=[v]_{i, j}$ for every $j$. Therefore:

$$
\begin{aligned}
G_{k}\left(v_{t}\right)= & \sum_{i \in I_{1} \backslash I_{2}} \frac{1}{k} f\left(a_{n} \sum_{j=1}^{k-1} \frac{1}{k}\left[v_{t}\right]_{i, j}\right)+\sum_{i \in I_{2} \backslash I_{1}} \frac{1}{k} f\left(a_{n} \sum_{j=1}^{k-1} \frac{1}{k}\left[v_{t}\right]_{i, j}\right) \\
& +\sum_{i \notin I_{1} \Delta I_{2}} \frac{1}{k} f\left(a_{n} \sum_{j=1}^{k-1} \frac{1}{k}[v]_{i, j}\right)=\sum_{i \in I_{1} \backslash I_{2}} \frac{1}{k} f\left(a_{n} \sum_{j=1}^{k-1} \frac{1}{k}[v]_{i, j}+\frac{a_{n}}{k}\left(\frac{j_{0}}{k}\right)^{n-2} t\right) \\
& +\sum_{i \in I_{2} \backslash I_{1}} \frac{1}{k} f\left(a_{n} \sum_{j=1}^{k-1} \frac{1}{k}[v]_{i, j}-\frac{a_{n}}{k}\left(\frac{j_{0}}{k}\right)^{n-2} t\right)+\sum_{i \notin I_{1} \Delta I_{2}} \frac{1}{k} f\left(a_{n} \sum_{j=1}^{k-1} \frac{1}{k}[v]_{i, j}\right) .
\end{aligned}
$$

The function $t \mapsto G_{k}\left(v_{t}\right)$ is twice continuously differentiable in $t=0$ (due to the smoothness of $f$ ), and:

$$
\left.\frac{\mathrm{d}^{2}}{\mathrm{~d} t^{2}} G_{k}\left(v_{t}\right)\right|_{t=0}=\frac{a_{n}^{2}}{k^{3}}\left(\frac{j_{0}}{k}\right)^{2(n-2)}\left[\sum_{i \in I_{1} \backslash I_{2}} f^{\prime \prime}\left(a_{n} \sum_{j=1}^{k-1} \frac{1}{k}[v]_{i, j}\right)+\sum_{i \in I_{2} \backslash I_{1}} f^{\prime \prime}\left(a_{n} \sum_{j=1}^{k-1} \frac{1}{k}[v]_{i, j}\right)\right]<0
$$


by the strict concavity of $f$; this is a contradiction, since $v$ is a minimizer for $G_{k}$.

Step 2. We claim that if $v \in Y_{k}^{N}$ takes only the values 0 and $a$, then $G_{k}(v) \geq G_{k}(\bar{v})$, where

$$
\bar{v}^{i, j}= \begin{cases}0 & i<N k \\ a & i \geq N k\end{cases}
$$

To this aim we can assume that

$$
E_{v}:=\left\{i \in \mathbb{Z}: \exists j v^{i+\hat{j}, j}=a, i+\hat{j}<N k\right\} \neq \emptyset
$$

(otherwise $v=\bar{v}$ ). Let $i_{0}=\min E_{v}$ and

$$
\begin{gathered}
j_{M}=\max \left\{j: \quad v^{i_{0}+\hat{j}, j}=a, i_{0}+\hat{j}<N k\right\}, \\
J_{M}=\left\{j: \hat{j}=\hat{j}_{M}, v^{i_{0}+\hat{j}, j}=a\right\}, \quad j_{0}=\min J_{M} .
\end{gathered}
$$

Then $v^{i_{0}+\hat{j}_{0}, j_{0}}=a$ and

$$
j<j_{0} \quad \Rightarrow \quad v^{i_{0}+\hat{j}_{0}, j}=0 .
$$

Indeed, if we had $j<j_{0}$ with the property $v^{i_{0}+\hat{j}_{0}, j}=a$, then we would have $\hat{j}>\hat{j}_{0}=\hat{j}_{M}$, and $N k>i_{0}+\hat{j}_{0}=$ $i_{0}-l+\hat{j}$, with $l=\hat{j}-\hat{j}_{0}>0$; therefore $v^{i_{0}-l+\hat{j}, j}=a$, and $i_{0}-l \in E$, which contrasts with the definition of $i_{0}$.

Denote by $w$ the function obtained by modifying $v$ in $\left(i_{0}+\hat{j}, j\right)$ for $j \in J_{M}$ :

$$
w^{i_{0}+\hat{j}, j}=0 \quad \text { for } j \in J_{M}, \quad w^{i, j}=v^{i, j} \quad \text { otherwise. }
$$

We want to show that

$$
G_{k}(v) \geq G_{k}(w) .
$$

Note that in the sum over $i$ defining $G_{k}(v)$ the terms $v^{i_{0}+\hat{j}, j}\left(j \in J_{M}\right)$ appear only if $i=i_{0}$ or $i=i_{0}+2 \hat{j}_{0}$. Accordingly, let us write $k G_{k}(v)$ as:

$$
k G_{k}(v)=f(q+\delta)+f(p)+\sum_{i \notin\left\{i_{0}, i_{0}+2 \hat{j}_{0}\right\}} f\left(\sum_{j} \frac{a_{n}}{k}[v]_{i, j}\right),
$$

where:

$$
q=\sum_{j \notin J_{M}} \frac{a_{n}}{k}[v]_{i_{0}, j}, \quad \delta=\sum_{j \in J_{M}} \frac{a_{n} a}{k}\left(\frac{j}{k}\right)^{n-2}, \quad p=\sum_{j=0}^{k-1} \frac{a_{n}}{k}[v]_{i_{0}+2 \hat{j}_{0}, j} .
$$

An analogous splitting can be written for $k G_{k}(w)$ : clearly, the last term is the same as in (5.7). Thus:

$$
k G_{k}(v)-k G_{k}(w)=f(q+\delta)-f(q)-[f(p+\delta)-f(p)] .
$$

By the definition of $j_{0}$ it turns out that:

$$
q=\sum_{j<j_{0}} \frac{a_{n}}{k}[v]_{i_{0}, j} .
$$

Moreover, if $j<j_{0}$ then $\hat{j} \geq \hat{j}_{0}$; the monotonicity in the first variable and (5.5) yield:

$$
v^{i_{0}+2 \hat{j}_{0}-\hat{j}, j} \leq v^{i_{0}+\hat{j}_{0}, j}=0 .
$$

Hence, $j<j_{0}$ implies that $v^{i_{0}+2 \hat{j}_{0}-\hat{j}, j}=0$, so that

$$
j<j_{0} \quad \Rightarrow \quad[v]_{i_{0}+2 \hat{j}_{0}, j}=v^{i_{0}+2 \hat{j}_{0}+\hat{j}, j}-v^{i_{0}+2 \hat{j}_{0}-\hat{j}, j} \geq v^{i_{0}+\hat{j}, j}=[v]_{i_{0}, j} .
$$


Therefore

$$
p \geq \sum_{j<j_{0}} \frac{a_{n}}{k}[v]_{i_{0}+2 \hat{j}_{0}, j} \geq q .
$$

Note now that the concavity of $f$ implies:

$$
q \leq p \quad \Rightarrow \quad f(q+\delta)-f(q) \geq f(p+\delta)-f(p)
$$

This, together with (5.8), proves (5.6). If $E_{w}$ is not empty, it has a minimum strictly greater than $i_{0}=\min E_{v}$. A finite iteration of the above argument proves the claim.

Step 3. We have shown that for every $N>0, \inf _{Y_{k}^{N}} G_{k}=G_{k}(\bar{v})$, where $\bar{v}$ does not depend on $N$. Then $\inf _{Y_{k}} G_{k}=\inf _{N>0} \inf _{Y_{k}^{N}} G_{k}=G_{k}(\bar{v})$. Let us estimate $G_{k}(\bar{v})$, where, by translation, we can suppose $\bar{v}$ given by

$$
\bar{v}^{i, j}= \begin{cases}0 & i<0 \\ a & i \geq 0\end{cases}
$$

We have

$$
G_{k}(\bar{v}) \geq 2 \sum_{i=-k}^{-1} \frac{1}{k} f\left(a_{n} \sum_{\substack{j=1 \\ i+\hat{j} \geq 0}}^{k-1} \frac{a}{k}\left(\frac{j}{k}\right)^{n-2}\right)=2 \sum_{i=-k}^{-1} \frac{1}{k} f\left(\frac{a_{n} a}{k} \sum_{j=1}^{\hat{i}}\left(\frac{j}{k}\right)^{n-2}\right),
$$

where $\hat{i}$ denotes the integer part of $\sqrt{k^{2}-i^{2}}$. Fix $\sigma>0$; then for $k \geq 1 / \sigma$ :

$$
\begin{aligned}
\frac{1}{k} \sum_{j=1}^{\hat{i}}\left(\frac{j}{k}\right)^{n-2} & \geq \frac{1}{k} \sum_{j=1}^{\hat{i}+1}\left(\frac{j}{k}\right)^{n-2}-\sigma \geq \int_{0}^{(\hat{i}+1) / k} t^{n-2} \mathrm{~d} t-\sigma \\
& \geq \int_{0}^{\sqrt{1-(i / k)^{2}}} t^{n-2} \mathrm{~d} t-\sigma=\frac{1}{n-1}\left(\sqrt{1-(i / k)^{2}}\right)^{n-1}-\sigma
\end{aligned}
$$

Then

$$
G_{k}(\bar{v}) \geq 2 \sum_{i=1}^{k} \frac{1}{k} f\left(a_{n} a\left[\frac{1}{n-1}\left(\sqrt{1-(i / k)^{2}}\right)^{n-1}-\sigma\right]\right) .
$$

Thus, by the definition of the Riemann integral as the limit of the Riemann sums, we have:

$$
\liminf _{k \rightarrow+\infty} \inf _{Y_{k}} G_{k} \geq 2 \int_{0}^{1} f\left(\frac{\omega_{n-1}}{\omega_{n}} a\left(\sqrt{1-t^{2}}\right)^{n-1}-a_{n} a \sigma\right) \mathrm{d} t .
$$

By the arbitrariness of $\sigma$ we conclude that:

$$
\liminf _{k \rightarrow+\infty} \inf _{Y_{k}} G_{k} \geq 2 \int_{0}^{1} f\left(\frac{\omega_{n-1}}{\omega_{n}} a\left(\sqrt{1-t^{2}}\right)^{n-1}\right) \mathrm{d} t .
$$

Step 4. We now relate $G$ with the discrete functionals $G_{k}$.

Let $v \in X$ : then there exist $\xi_{0}<\xi_{1}$ such that $v(\xi, \eta)=0$ if $\xi \leq \xi_{0}$ and $v(\xi, \eta)=a$ if $\xi \geq \xi_{1}$. Extend $v$ on $\mathbb{R}^{2}$ with $v(\xi, \eta)=a$ if $\xi \geq \xi_{1}$ and $v(\xi, \eta)=0$ otherwise outside $\mathbb{R} \times[0,1]$. If $\left(\varrho_{\tau}\right)_{\tau>0}$ is a family of mollifiers, it is easy to see that $v * \varrho_{\tau} \in X \cap C^{\infty}$, and $G\left(v * \varrho_{\tau}\right) \rightarrow G(v)$ as $\tau \rightarrow 0$.

Then, given $\sigma>0$ there exists $v_{\sigma} \in X \cap C^{\infty}$ such that

$$
\inf _{X} G \geq G\left(v_{\sigma}\right)-\sigma
$$


Assume that $v_{\sigma}(\xi, \eta)=0$ if $\xi \leq-N$ and $v_{\sigma}(\xi, \eta)=a$ if $\xi \geq N$, for a suitable $N \in \mathbb{N}$. Since $v_{\sigma}$ is uniformly continuous, there exists $\delta>0$ such that

$$
\left|(\xi, \eta)-\left(\xi^{\prime}, \eta^{\prime}\right)\right|<\delta \Rightarrow\left|v_{\sigma}(\xi, \eta)-v_{\sigma}\left(\xi^{\prime}, \eta^{\prime}\right)\right|<\sigma / N .
$$

Fix $k \in \mathbb{N}$, and define $Q_{k}^{i, j}=[i / k,(i+1) / k) \times[j / k,(j+1) / k)$, for $i \in \mathbb{Z}$ and $j \in\{1, \ldots, k-1\}$. If $(\xi, \eta) \in Q_{k}^{i, j}$ then

Note that

$$
\frac{i+\widehat{(j+1)}}{k} \leq \xi+\sqrt{1-\eta^{2}} \leq \frac{i+\hat{j}+2}{k}
$$

$$
0 \leq \frac{\hat{j}}{k}-\frac{\widehat{(j+1)}}{k} \leq \frac{1+\sqrt{2 k-1}}{k} \stackrel{k}{\rightarrow} 0
$$

Then there exists $k_{\delta}$ such that for every $k \geq k_{\delta}, i \in \mathbb{Z}, j \in\{1, \ldots, k-1\}$ :

$$
(\xi, \eta) \in Q_{k}^{i, j},\left(\xi^{\prime}, \eta^{\prime}\right) \in Q_{k}^{i+\hat{j}, j} \Rightarrow\left|\left(\xi+\sqrt{1-\eta^{2}}, \eta\right)-\left(\xi^{\prime}, \eta^{\prime}\right)\right|<\delta .
$$

It follows that

Analogously, we have

$$
(\xi, \eta) \in Q_{k}^{i, j} \Rightarrow\left|v_{\sigma}\left(\xi+\sqrt{1-\eta^{2}}, \eta\right)-f_{Q_{k}^{i+\hat{\jmath}, j}} v_{\sigma} \mathrm{d} x\right|<\sigma / N .
$$

$$
(\xi, \eta) \in Q_{k}^{i, j} \Rightarrow\left|v_{\sigma}\left(\xi-\sqrt{1-\eta^{2}}, \eta\right)-f_{Q_{k}^{i-\hat{\jmath}, j}} v_{\sigma} \mathrm{d} x\right|<\sigma / N .
$$

For every $k \in \mathbb{N}$ and $(i, j) \in \mathbb{Z} \times\{1, \ldots, k-1\}$ let

$$
w_{\sigma}^{i, j}=f_{Q_{k}^{i, j}} v_{\sigma} \mathrm{d} x
$$

Clearly, $w_{\sigma}=\left(w_{\sigma}^{i, j}\right) \in Y_{k}$. For every $i, j$ and $(\xi, \eta) \in Q_{k}^{i, j}$

$$
\left[v_{\sigma}\left(\xi+\sqrt{1-\eta^{2}}, \eta\right)-v_{\sigma}\left(\xi-\sqrt{1-\eta^{2}}, \eta\right)\right] \eta^{n-2} \geq\left[w_{\sigma}\right]_{i, j}-2 \sigma / N .
$$

This implies that for every $i \in \mathbb{Z}$ and $\xi \in[i / k,(i+1) / k)$

$$
\int_{0}^{1}\left[v_{\sigma}\left(\xi+\sqrt{1-\eta^{2}}, \eta\right)-v_{\sigma}\left(\xi-\sqrt{1-\eta^{2}}, \eta\right)\right] \eta^{n-2} \mathrm{~d} \eta \geq \frac{1}{k} \sum_{j=1}^{k-1}\left[w_{\sigma}\right]_{i, j}-2 \sigma / N .
$$

Finally, since $f$ (extended with value 0 on $\mathbb{R}^{-}$) is Lipschitz continuous with Lipschitz constant 1, we have:

$$
G\left(v_{\sigma}\right) \geq \sum_{i=-(N+1) k}^{(N+1) k} \frac{1}{k} f\left(a_{n} \sum_{j=1}^{k-1} \frac{1}{k}\left[w_{\sigma}\right]_{i, j}-\frac{2 \sigma a_{n}}{N}\right) \geq G_{k}\left(w_{\sigma}\right)-2 \sigma a_{n}(2 N+3) / N .
$$

By (5.10), and the arbitrariness of $\sigma$, we obtain

$$
\inf _{X} G \geq \liminf _{k \rightarrow+\infty} \inf _{Y_{k}} G_{k}
$$

and this, by (5.9) of Step 3, concludes the proof. 


\section{Estimate from Below of the LOWER $\Gamma$-Limit}

We collect here the results of Sections 4 and 5 , proving the lower bound $F^{\prime} \geq \mathcal{F}$. Recall that, by Corollary 4.2 , if $F^{\prime}(u)<+\infty$ then $u \in G B V(\Omega)$.

Theorem 6.1. For every $u \in G B V(\Omega)$

$$
F^{\prime}(u) \geq \int_{\Omega}|\nabla u(x)| \mathrm{d} x+\int_{S_{u}} \vartheta\left(\left|u^{+}(x)-u^{-}(x)\right|\right) \mathrm{d} \mathcal{H}^{n-1}+\left|D^{c} u\right|(\Omega) .
$$

Proof. Let $u \in B V(\Omega)$ and let $A$ be an open subset of $\Omega$. From Proposition 4.7 we have

$$
F^{\prime}(u, A) \geq \int_{A}|\nabla u(x)| \mathrm{d} x, \quad F^{\prime}(u, A) \geq\left|D^{c} u\right|(A)
$$

moreover, Corollary 5.3 and Propositions 5.5, 5.6 and 5.7 give

$$
F^{\prime}(u, A) \geq \int_{S_{u} \cap A} \vartheta\left(\left|u^{+}(x)-u^{-}(x)\right|\right) \mathrm{d} \mathcal{H}^{n-1} .
$$

Apply now Corollary 2.8 (which clearly extends to a finite number of measures $\lambda_{i}$ ), with $\lambda_{1}=\mathcal{L}^{n}, \lambda_{2}=$ $\mathcal{H}^{n-1}\left\llcorner S_{u}\right.$ and $\lambda_{3}=\left|D^{c} u\right|$. Therefore $F^{\prime}(u) \geq \mathcal{F}(u)$.

Let us now consider the case $u \in G B V(\Omega)$. We can find a positive infinitesimal sequence $\left(\varepsilon_{j}\right)$ and a sequence $\left(u_{j}\right)$ in $W^{1,1}(\Omega)$ converging to $u$ in $L^{1}(\Omega)$ and such that $F_{\varepsilon_{j}}\left(u_{j}\right) \stackrel{j}{\rightarrow} F^{\prime}(u)$. Define $u_{j}^{T}=(-T) \vee u_{j} \wedge T$, and $u^{T}=(-T) \vee u \wedge T$. Since $u_{j}^{T} \rightarrow u^{T}$ in $L^{1}(\Omega)$, and $u^{T} \in B V(\Omega)$, we have

$F^{\prime}(u)=\liminf _{j \rightarrow+\infty} F_{\varepsilon_{j}}\left(u_{j}\right) \geq \liminf _{j \rightarrow+\infty} F_{\varepsilon_{j}}\left(u_{j}^{T}\right) \geq \int_{\Omega}\left|\nabla u^{T}(x)\right| \mathrm{d} x+\int_{S_{u} \cap \Omega} \vartheta\left(\left|\left(u^{T}\right)^{+}(x)-\left(u^{T}\right)^{-}(x)\right|\right) \mathrm{d} \mathcal{H}^{n-1}+\left|D^{c} u^{T}\right|(\Omega)$.

We conclude by taking the limit as $j \rightarrow+\infty$ and recalling the definitions of $\nabla u, u^{ \pm}$and $\left|D^{c} u\right|($ see $(2.2),(2.3)$ and $(2.4))$.

\section{Estimate From AbOve of the Upper $\Gamma$-Limit}

In this last section we conclude the proof of Theorem 3.1 by proving the upper estimate for the $\Gamma$-limit (Prop. 7.3). The right bound will be first obtained for a suitable dense subset of $S B V(\Omega)$ : let $\mathcal{W}(\Omega)$ be the space of all functions $w \in S B V(\Omega)$ satisfying the following properties:

i) $\mathcal{H}^{n-1}\left(\bar{S}_{w} \backslash S_{w}\right)=0$;

ii) $\bar{S}_{w}$ is the intersection of $\Omega$ with the union of a finite member of $(n-1)$-dimensional simplexes;

iii) $w \in W^{k, \infty}\left(\Omega \backslash \bar{S}_{w}\right)$ for every $k \in \mathbb{N}$.

From [17] we get the density property of $\mathcal{W}(\Omega)$ we need; we recall that $S B V^{2}(\Omega)=\{u \in S B V(\Omega):|\nabla u| \in$ $\left.L^{2}(\Omega), \mathcal{H}^{n-1}\left(S_{u}\right)<+\infty\right\}$.

Theorem 7.1 [17] Th. 3.1). Assume that $\partial \Omega$ is Lipschitz. Let $u \in S B V^{2}(\Omega) \cap L^{\infty}(\Omega)$. Then there exists a sequence $\left(w_{j}\right)$ in $\mathcal{W}(\Omega)$ such that $w_{j} \rightarrow u$ strongly in $L^{1}(\Omega), \nabla w_{j} \rightarrow \nabla u$ strongly in $L^{2}\left(\Omega, \mathbb{R}^{n}\right), \lim _{\sup _{h}}\left\|w_{j}\right\|_{\infty} \leq$ $\|u\|_{\infty}$ and

$$
\limsup _{j \rightarrow+\infty} \int_{S_{w_{j}}} \phi\left(w_{j}^{+}, w_{j}^{-}, \nu_{w_{j}}\right) \mathrm{d} \mathcal{H}^{n-1} \leq \int_{S_{u}} \phi\left(u^{+}, u^{-}, \nu_{u}\right) \mathrm{d} \mathcal{H}^{n-1}
$$

for every upper semicontinuous function $\phi$ such that $\phi(a, b, \nu)=\phi(b, a,-\nu)$ whenever $a, b \in \mathbb{R}$ and $\nu \in S^{n-1}$. 
Lemma 7.2. Let $g \in L^{1}(\Omega)$ and let $A$ be an open subset of $\Omega$. Then

$$
\lim _{\varepsilon \rightarrow 0} \frac{1}{\varepsilon} \int_{A} f\left(\varepsilon f_{B_{\varepsilon}(x) \cap \Omega} g(y) \mathrm{d} y\right) \mathrm{d} x=\int_{A} g(x) \mathrm{d} x .
$$

Proof. Let $g_{\varepsilon}(x)=f_{B_{\varepsilon}(x) \cap \Omega} g(y) \mathrm{d} y$. Since $g \in L^{1}(\Omega)$, from Lebesgue's differentation Theorem $g_{\varepsilon}$ converges to $g$ in $L^{1}(\Omega)$ and a.e. in $\Omega$. We can now conclude by the dominated convergence Theorem, recalling that $f(t) \leq t$ and $f(t) / t \rightarrow 1$ as $t \rightarrow 0^{+}$.

Proposition 7.3. Let $u \in G B V(\Omega)$; then

$$
F^{\prime \prime}(u) \leq \int_{\Omega}|\nabla u(x)| \mathrm{d} x+\int_{S_{u}} \vartheta\left(\left|u^{+}(x)-u^{-}(x)\right|\right) \mathrm{d} \mathcal{H}^{n-1}+\left|D^{c} u\right|(\Omega)
$$

where $\vartheta$ is as in Theorem 3.1 .

Proof.

Step 1. Assume first that $u$ is in the space $\mathcal{W}(\Omega)$ introduced above. In particular $S_{u}$ is contained in the union of a finite collection $K_{1}, \ldots, K_{m}$ of $(n-1)$-dimensional simplexes. Since the upper $\Gamma$-limit of $\left(F_{\varepsilon}\right)$ coincides with the upper $\Gamma$-limit of $\left(\bar{F}_{\varepsilon}\right)$, we have $F^{\prime \prime}(u) \leq \lim \sup _{\varepsilon} \bar{F}_{\varepsilon}(u)$ : we intend to estimate this limit.

Fix $\sigma>0$. Let $S$ be the union of the relative boundaries of $K_{i}$ (note that $\mathcal{H}^{n-1}(S)=0$ ). Define

$$
\Omega_{\sigma}=\{x \in \Omega: d(x, \partial \Omega)>\sigma, d(x, S)>\sigma\} .
$$

By the integral representation of $\bar{F}_{\varepsilon}$ given in Proposition 3.6, and by the same argument used in Remark 3.7, we get:

$$
\bar{F}_{\varepsilon}(u) \leq \bar{F}_{\varepsilon}\left(u, \Omega_{\sigma}\right)+\frac{\omega_{n}}{\gamma}|D u|\left(A_{\varepsilon, \sigma}\right),
$$

where $A_{\varepsilon, \sigma}=\left\{x \in \Omega: d\left(x, \Omega \backslash \Omega_{\sigma}\right)<\varepsilon\right\}$. Clearly, $\lim _{\sigma} \lim \sup _{\varepsilon}|D u|\left(A_{\varepsilon, \sigma}\right)=0$. To deal with $\bar{F}_{\varepsilon}\left(u, \Omega_{\sigma}\right)$ it will not be restrictive to assume $m=1$ and $K:=K_{1} \subseteq\left\{x \in \mathbb{R}^{n}: x_{1}=0\right\}$.

By the subadditivity of $f$, if $\varepsilon<\sigma$ :

$$
\begin{aligned}
\bar{F}_{\varepsilon}\left(u, \Omega_{\sigma}\right) & \leq \frac{1}{\varepsilon} \int_{\Omega_{\sigma}} f\left(\varepsilon f_{B_{\varepsilon}(x)}|\nabla u(y)| \mathrm{d} y+\frac{1}{\omega_{n} \varepsilon^{n-1}}\left|D^{s} u\right|\left(B_{\varepsilon}(x)\right)\right) \mathrm{d} x \\
& \leq \frac{1}{\varepsilon} \int_{\Omega_{\sigma}} f\left(\varepsilon f_{B_{\varepsilon}(x)}|\nabla u(y)| \mathrm{d} y\right) \mathrm{d} x+\frac{1}{\varepsilon} \int_{\Omega_{\sigma}} f\left(\frac{\left|D^{s} u\right|\left(B_{\varepsilon}(x)\right)}{\omega_{n} \varepsilon^{n-1}}\right) \mathrm{d} x .
\end{aligned}
$$

In view of Lemma 7.2, the first of these last two terms tends to $\int_{\Omega_{\sigma}}|\nabla u| \mathrm{d} x$. Denote by $R_{\varepsilon}$ the other integral term, and let $K_{\varepsilon}=\left\{x \in \mathbb{R}^{n}: d(x, K)<\varepsilon\right\}$. Since $u$ is smooth outside $\left\{x_{1}=0\right\}$, we have:

$$
R_{\varepsilon}=\frac{1}{\varepsilon} \int_{\Omega_{\sigma} \cap K_{\varepsilon}} f\left(\frac{1}{\omega_{n} \varepsilon^{n-1}}\left|D^{s} u\right|\left(B_{\varepsilon}(x)\right)\right) \mathrm{d} x .
$$

If $K_{\varepsilon, \sigma} \subseteq \mathbb{R}^{n-1}$ denotes the projection of $\Omega_{\sigma} \cap K_{\varepsilon}$ onto $\left\{x_{1}=0\right\}$, it turns out that $\Omega_{\sigma} \cap K_{\varepsilon}$ is contained in $(-\varepsilon, \varepsilon) \times K_{\varepsilon, \sigma}$, which is a subset of $\{x \in \Omega: d(x, \partial \Omega)>\varepsilon\}$ for $\varepsilon$ sufficiently small. Then:

$$
R_{\varepsilon} \leq \frac{1}{\varepsilon} \int_{K_{\varepsilon, \sigma}} \mathrm{d} y \int_{-\varepsilon}^{\varepsilon} f\left(\frac{1}{\omega_{n} \varepsilon^{n-1}}\left|D^{s} u\right|\left(B_{\varepsilon}(s, y)\right)\right) \mathrm{d} s .
$$


Set

$$
h(x)=u^{+}(x)-u^{-}(x), \quad x \in \Omega \cap\left\{x_{1}=0\right\} .
$$

Since $u \in \mathcal{W}(\Omega)$, the function $h$ is continuous, hence uniformly continuous on any compact subset of $\Omega \cap\left\{x_{1}=0\right\}$. Then, for $\varepsilon$ sufficiently small:

$$
\left|D^{s} u\right|\left(B_{\varepsilon}(s, y)\right)=\int_{B_{\varepsilon}(s, y) \cap\left\{x_{1}=0\right\}}|h| \mathrm{d} \mathcal{H}^{n-1} \leq \omega_{n-1}\left(\sqrt{\varepsilon^{2}-s^{2}}\right)^{n-1}(|h(0, y)|+\sigma) .
$$

Therefore

$$
R_{\varepsilon} \leq \frac{1}{\varepsilon} \int_{K_{\varepsilon, \sigma}}\left(\int_{-\varepsilon}^{\varepsilon} f\left(\frac{\omega_{n-1}}{\omega_{n}}\left(\sqrt{1-(s / \varepsilon)^{2}}\right)^{n-1}(|h(0, y)|+\sigma)\right) \mathrm{d} s\right) \mathrm{d} y .
$$

By the change of variable $t=s / \varepsilon$ we deduce that

$$
\limsup _{\varepsilon \rightarrow 0} R_{\varepsilon} \leq \int_{\Omega \cap\left\{x_{1}=0\right\}}\left(\int_{-1}^{1} f\left(\frac{\omega_{n-1}}{\omega_{n}}\left(\sqrt{1-t^{2}}\right)^{n-1}(|h(z)|+\sigma)\right) \mathrm{d} t\right) \mathrm{d} \mathcal{H}^{n-1}(z) .
$$

Now we conclude by taking the limit as $\sigma \rightarrow 0$, and noting that $h(z)$ vanishes $\mathcal{H}^{n-1}$-a.e. outside $S_{u}$.

Step 2. In the case $u \in S B V^{2}(\Omega) \cap L^{\infty}(\Omega)$, we can apply Theorem 7.1, with $\phi(a, b, \nu)=\vartheta(|a-b|)$. Then there exists a sequence $w_{j} \rightarrow u$ in $L^{1}(\Omega)$, with $w_{j} \in \mathcal{W}(\Omega)$, such that $\nabla w_{j} \rightarrow \nabla u$ in $L^{2}\left(\Omega, \mathbb{R}^{n}\right)$ and

$$
\limsup _{j \rightarrow+\infty} \int_{S_{w_{j}}} \vartheta\left(\left|w_{j}^{+}(x)-w_{j}^{-}(x)\right|\right) \mathrm{d} \mathcal{H}^{n-1} \leq \int_{S_{u}} \vartheta\left(\left|u^{+}(x)-u^{-}(x)\right|\right) \mathrm{d} \mathcal{H}^{n-1}
$$

thus, by the lower semicontinuity of $F^{\prime \prime}$ and by Step 1 ,

$$
F^{\prime \prime}(u) \leq \liminf _{j \rightarrow+\infty} F^{\prime \prime}\left(w_{j}\right) \leq \int_{\Omega}|\nabla u(x)| \mathrm{d} x+\int_{S_{u}} \vartheta\left(\left|u^{+}(x)-u^{-}(x)\right|\right) \mathrm{d} \mathcal{H}^{n-1} .
$$

Using now the relaxation Theorem 2.4, we have

$$
F^{\prime \prime}(u) \leq \int_{\Omega}|\nabla u(x)| \mathrm{d} x+\int_{S_{u}} \vartheta\left(\left|u^{+}(x)-u^{-}(x)\right|\right) \mathrm{d} \mathcal{H}^{n-1}+\left|D^{c} u\right|(\Omega)
$$

for every $u \in B V(\Omega)$. Finally, by a truncation argument and again the lower semicontinuity of $F^{\prime \prime}$ we obtain the desired inequality in $G B V(\Omega)$.

\section{REFERENCES}

[1] R. Alicandro, A. Braides and M.S. Gelli, Free-discontinuity problems generated by singular perturbation. Proc. Roy. Soc. Edinburgh Sect. A 6 (1998) 1115-1129.

[2] R. Alicandro, A. Braides and J. Shah, Free-discontinuity problems via functionals involving the $L^{1}$-norm of the gradient and their approximations. Interfaces and free boundaries 1 (1999) 17-37.

[3] R. Alicandro and M.S. Gelli, Free discontinuity problems generated by singular perturbation: the $n$-dimensional case. Proc. Roy. Soc. Edinburgh Sect. A 130 (2000) 449-469.

[4] L. Ambrosio, A compactness theorem for a new class of functions of bounded variation. Boll. Un. Mat. Ital. B 3 (1989) $857-881$.

[5] L. Ambrosio, N. Fusco and D. Pallara, Functions of Bounded Variation and Free Discontinuity Problems. Oxford University Press (2000).

[6] L. Ambrosio and V.M. Tortorelli, Approximation of functionals depending on jumps by elliptic functionals via $\Gamma$-convergence. Comm. Pure Appl. Math. XLIII (1990) 999-1036. 
[7] L. Ambrosio and V.M. Tortorelli, On the approximation of free discontinuity problems. Boll. Un. Mat. Ital. B (7) VI (1992) $105-123$.

[8] G. Bouchitté, A. Braides and G. Buttazzo, Relaxation results for some free discontinuity problems. J. Reine Angew. Math. 458 (1995) 1-18.

[9] B. Bourdin and A. Chambolle, Implementation of an adaptive finite-element approximation of the Mumford-Shah functional. Numer. Math. 85 (2000) 609-646.

[10] A. Braides. Approximation of free-discontinuity problems. Lect. Notes Math. 1694, Springer Verlag, Berlin (1998).

[11] A. Braides and A. Garroni, On the non-local approximation of free-discontinuity problems. Comm. Partial Differential Equations 23 (1998) 817-829.

[12] A. Braides and G. Dal Maso, Non-local approximation of the Mumford-Shah functional. Calc. Var. 5 (1997) 293-322.

[13] A. Chambolle and G. Dal Maso, Discrete approximation of the Mumford-Shah functional in dimension two. ESAIM: M2AN 33 (1999) 651-672.

[14] G. Cortesani, Sequence of non-local functionals which approximate free-discontinuity problems. Arch. Rational Mech. Anal. 144 (1998) 357-402.

[15] G. Cortesani, A finite element approximation of an image segmentation problem. Math. Models Methods Appl. Sci. 9 (1999) $243-259$.

[16] G. Cortesani and R. Toader, Finite element approximation of non-isotropic free-discontinuity problems. Numer. Funct. Anal. Optim. 18 (1997) 921-940.

[17] G. Cortesani and R. Toader, A density result in SBV with respect to non-isotropic energies. Nonlinear Anal. 38 (1999) $585-604$.

[18] G. Cortesani and R. Toader, Nonlocal approximation of nonisotropic free-discontinuity problems. SIAM J. Appl. Math. 59 (1999) 1507-1519.

[19] G. Dal Maso, An Introduction to $\Gamma$-Convergence. Birkhäuser, Boston (1993).

[20] E. De Giorgi. Free discontinuity problems in calculus of variations, in Frontiers in pure and applied mathematics. A collection of papers dedicated to Jacques-Louis Lions on the occasion of his sixtieth birthday. June 6-10, Paris 1988, Robert Dautray, Ed., Amsterdam, North-Holland Publishing Co. (1991) 55-62.

[21] L. Lussardi and E. Vitali, Non-local approximation of free-discontinuity functionals with linear growth: the one-dimensional case. Ann. Mat. Pura Appl. (to appear).

[22] M. Morini, Sequences of singularly perturbed functionals generating free-discontinuity problems. SIAM J. Math. Anal. 35 (2003) 759-805.

[23] M. Negri, The anisotropy introduced by the mesh in the finite element approximation of the Mumford-Shah functional. Numer. Funct. Anal. Optim. 20 (1999) 957-982.

[24] S. Osher and J.A. Sethian, Fronts propagating with curvature-dependent speed: algorithms based on Hamilton-Jacobi formulations. J. Comput. Phys. 79 (1988) 12-49.

[25] J. Shah, A common framework for curve evolution, segmentation and anisotropic diffusion, in IEEE conference on computer vision and pattern recognition (1996).

[26] J. Shah, Uses of elliptic approximations in computer vision. In R. Serapioni and F. Tomarelli, editors, Progress in Nonlinear Differential Equations and Their Applications 25 (1996).

[27] L. Simon, Lectures on Geometric Measure Theory. Centre for Mathematical Analysis, Australian National University (1984). 